Sagun Chanillo · Carlos E. Kenig

\title{
Weak uniqueness and partial regularity for the composite membrane problem
}

Received February 7, 2007 and in revised form April 6, 2007

\begin{abstract}
We study the composite membrane problem in all dimensions. We prove that the minimizing solutions exhibit a weak uniqueness property which under certain conditions can be turned into a full uniqueness result. Next we study the partial regularity of the solutions to the EulerLagrange equation associated to the composite problem and also the regularity of the free boundary for solutions to the Euler-Lagrange equations.
\end{abstract}

Keywords. Free boundary, partial regularity, monotonicity formula, composite membrane, uniqueness

\section{Introduction}

Our main concern will be the physical problem proposed in [CGI $\left.{ }^{+} 00\right]$ which can be stated as:

Problem (P). Build a body of prescribed shape out of given materials of varying density, in such a way that the body has prescribed mass and so that the basic frequency (with fixed boundary) is as small as possible.

By virtue of Theorem 13 in [CGI ${ }^{+} 00$ this problem can be converted into the following minimization problem. Given a bounded domain $\Omega \subset \mathbb{R}^{n}$ with smooth boundary, fix $\alpha>0$ and $A \in[0,|\Omega|]$. For any measurable subset $D \subset \Omega$, denote by $\lambda_{\Omega}(\alpha, D)$ the first Dirichlet eigenvalue for the problem

$$
-\Delta u+\alpha \chi_{D} u=\lambda_{\Omega}(\alpha, D) u \quad \text { in } \Omega, \quad u=0 \quad \text { on } \partial \Omega .
$$

Define

$$
\Lambda_{\Omega}(\alpha, A)=\inf _{D \subset \Omega,|D|=A} \lambda_{\Omega}(\alpha, D) .
$$

A minimizer $D$ to $(1.2)$ will be called an optimal configuration for the data $(\Omega, \alpha, A)$. For this $D$ we denote the associated eigenfunction solution to $(1.1)$ by $u$. The pair $(u, D)$

S. Chanillo: Department of Mathematics, Rutgers University, Piscataway, NJ 08854, USA; e-mail: chanillo@math.rutgers.edu

C. E. Kenig: Department of Mathematics, University of Chicago, Chicago, IL 60637, USA; e-mail: cek@math.uchicago.edu

Mathematics Subject Classification (2000): 35R35, 35J60, 35B65 
will be called an optimal pair solution to the composite problem or for short a solution to the composite problem.

A variational formulation of our problem is also possible and is given by (see $\left[\mathrm{CGI}^{+} 00\right]$ )

$$
\Lambda_{\Omega}(\alpha, A)=\inf _{u \in H_{0}^{1}(\Omega),|D|=A,\|u\|_{2}=1} \int_{\Omega}\left(|\nabla u|^{2}+\alpha \chi_{D} u^{2}\right) .
$$

Theorem 1 in $\left[\mathrm{CGI}^{+} 00\right]$ establishes the basic properties of the existence and regularity of optimal pairs.

Theorem 1.1 ([CGI $\left.\left.{ }^{+} 00\right]\right)$. For any $\alpha>0$ and $A \in[0,|\Omega|]$, there exists an optimal pair $(u, D)$. Moreover, it has the following properties:

(a) $u \in C^{1, \gamma}(\bar{\Omega}) \cap H^{2}(\bar{\Omega})$ for every $\gamma<1$.

(b) $D$ is a sublevel set of $u$, that is, there exists $c \geq 0$ such that $D=\{u \leq c\}$.

(c) If $\alpha \neq \Lambda_{\Omega}(\alpha, A)$, then every level set $\{u=s\}$ has measure zero.

See Remark 2.2 for additional comments regarding (c). From Theorem 13 in [CGI ${ }^{+} 00$ ] we also know that the physical problem $(\mathrm{P})$ stated earlier is equivalent to the variational problem (1.3) provided

$$
\alpha<\Lambda_{\Omega}(\alpha, A) .
$$

In the following we shall always assume 1.47 . Now putting together Theorem 1.1 and the variational characterization (1.3) of the problem we see that the Euler-Lagrange equation of our problem is

$$
-\Delta u+\alpha \chi_{\{u \leq c\}} u=\Lambda_{\Omega}(\alpha, A) u \quad \text { in } \Omega, \quad u=0 \quad \text { on } \partial \Omega .
$$

In Section 2 we first turn to the problem of uniqueness of optimal pairs $(u, D)$. A principal result of $\left[\mathrm{CGI}^{+} 00\right]$ is that even in domains that exhibit symmetry, the optimal pair need not be unique, and in fact uniqueness is known without any assumptions only if $\Omega$ is the ball. Nevertheless, we establish that generically there is a sort of weak uniqueness.

Theorem 1.2 (Weak uniqueness). Assume (1.4). For almost every $A \in(0,|\Omega|)$, there exists $c>0$ such that for all optimizing pairs $\left(u_{i}, D_{i}\right)$,

$$
D_{i}=\left\{x: u_{i}(x) \leq c\right\}
$$

Thus though there is non-uniqueness in the problem, the level height where one must cut off the eigenfunction to get $D_{i}$ must generically be the same for all eigenfunctions.

Under additional assumptions, namely if eigenfunctions agree at one point to infinite order or if $\Omega$ is convex in $\mathbb{R}^{2}$ with additional assumptions, the assertion of weak uniqueness can be turned into a statement of true uniqueness. See, for example, Lemma2.9 and Theorem2.1 in Section 2.

In Section 3 we turn to the regularity of the free boundary $\mathcal{F}$, defined by

$$
\mathcal{F}=\{x: u(x)=c\} .
$$

We recall an initial result, Theorem 8 in [CGK00]: 
Theorem 1.3. Let $x_{0} \in \mathcal{F}$. Assume $\nabla u\left(x_{0}\right) \neq 0$, that is, $x_{0}$ is a regular point of the free boundary. Then there exists a ball $B\left(x_{0}, r\right)$ of radius $r>0$ centered at $x_{0}$, and $a$ real-analytic function $\phi\left(x_{1}, \ldots, x_{n-1}\right)$ such that

$$
\mathcal{F} \cap B\left(x_{0}, r\right)=\left\{\left(x_{1}, \ldots, x_{n}\right): x_{n}=\phi\left(x_{1}, \ldots, x_{n-1}\right)\right\} .
$$

That is, the free boundary in the neighborhood of a regular point is a hypersurface given by the graph of a real-analytic function.

Subsequently Blank [Bla04] performed a blow-up analysis in dimension 2 to classify the singular points of $\mathcal{F}$, that is, those points on $\mathcal{F}$ where $\nabla u=0$. This analysis in dimension 2 was completed by Shahgholian [Sha], who also obtained a condition that guarantees that the singular points of $\mathcal{F}$ in dimension 2 are isolated.

The free boundary problem for the composite problem can be easily converted to an equivalent problem (see e.g. [Sha] $)$ given by

$$
\Delta v=f \chi_{\{v \geq 0\}}-g \chi\{v \leq 0\}, \quad f, g \in C^{1, \gamma}, f>0, g<0, f+g<0 .
$$

Our main result concerning the structure of $\mathcal{F}$ in Section 3 is:

Theorem 1.4 (Structure of the free boundary of solutions $(1.7)$ ). For $\Omega \subset \mathbb{R}^{n}$, there is $a$ decomposition

$$
\mathcal{F}=\mathcal{F}_{0} \cup S_{v}^{1} \cup S_{v}^{2}
$$

where $S_{v}^{2}$ has Hausdorff dimension $\leq n-2, \mathcal{H}^{n-1}\left(S_{v}^{1}\right) \leq C$, and for all $x_{0} \in \mathcal{F}_{0}$, there exists a ball $B\left(x_{0}, r\right)$ such that $\mathcal{F} \cap B\left(x_{0}, r\right)$ is a hypersurface given by the graph of a real-analytic function.

The principal tool we use to perform our blow-up analysis and thereby get Theorem 1.4 is an energy functional introduced by Weiss [Wei98]. Set $\left(f \equiv f_{0}, g \equiv g_{0}\right)$

$$
W(r)=\frac{1}{r^{n+2}} \int_{B\left(x_{0}, r\right)}\left(|\nabla v|^{2}+2\left(f_{0} v^{+}+g_{0} v^{-}\right)\right)-\frac{2}{r^{n+3}} \int_{\partial B\left(x_{0}, r\right)} u^{2} .
$$

Weiss showed that $W(r)$ is increasing. We offer an alternative proof based in part on the Rellich-Pokhozhaev identity which explicitly shows that no structural assumptions are needed to get the monotonicity.

Next we proceed to classify the blow-up limits in the spirit of the paper by MonneauWeiss [MW07]. Two points are to be noted in contrast to [MW07]. First, in our case blowup limits are non-degenerate, and second, we have two types of blow-up limit solutions that are homogeneous of degree 2. This is already evident in the work in dimension 2 by Blank [Bla04] and Shahgholian [Sha].

Lastly, we address the question of $C^{1,1}$ bounds. In general such bounds are not available for the composite problem if we only analyze the Euler-Lagrange equation (1.7). Socalled cross solutions arise from homogeneous harmonic polynomials of degree 2 with corresponding failure of $C^{1,1}$ bounds in dimension 2, as has been exhibited by Andersson and Weiss [AW06] in the case $f \equiv-1, g \equiv 0$. The example of Andersson-Weiss can be easily extended to all dimensions by the addition of dummy variables. We show that the [AW06 construction extends to our setting (Remark 3.20. Our regularity result proved in Section 3 is (see Theorem 3.4, Definition 3.16 and Remark 3.19): 
Theorem 1.5. We have $\mathcal{F}=G \cup B$, where in $G$ we have pointwise $C^{1,1}$ bounds and $B$ has Hausdorff dimension $\leq n-2$.

It remains open whether proceeding from the variational problem instead of (1.7) allows one to get $C^{1,1}$ bounds. It is readily seen that global assumptions on the boundary of $\Omega$ do ensure that $C^{1,1}$ bounds and full regularity are achieved. A result of this type proved in Section 3 is (see Proposition 3.7):

Proposition 1.6. Assume $\Omega \subset \mathbb{R}^{2}$ has two axes of symmetry, where symmetry is defined in the sense of Theorem 4 in $\left[\mathrm{CGI}^{+} 00\right]$. Then the free boundary $\mathcal{F}$ is a real-analytic curve and $u \in C^{1,1}$.

\section{Uniqueness and weak uniqueness}

Our goal in this section is to prove Theorem 1.2 of the introduction. We shall also show that a weak uniqueness assertion as in Theorem 1.2 can be converted to a uniqueness assertion on convex domains with additional assumptions. Let $\Omega \subset \mathbb{R}^{n}$ be a bounded domain with $\partial \Omega$ smooth. For $\alpha>0, A \in[0,|\Omega|]$, and $D \subset \Omega$, let $\lambda_{\Omega}(\alpha, D)=\lambda$ be the lowest eigenvalue of

$$
\left\{\begin{array}{l}
-\Delta v+\alpha \chi_{D} v=\lambda v \quad \text { on } \Omega, \\
\left.v\right|_{\partial \Omega}=0 .
\end{array}\right.
$$

The variational characterization of (2.1) gives

$$
\lambda=\inf _{u \in H_{0}^{1}(\Omega)} \frac{\int_{\Omega}\left(|\nabla u|^{2}+\alpha \chi_{D} u^{2}\right)}{\int_{\Omega} u^{2}} .
$$

Lemma 2.1. There exists a unique minimizer $v \in H_{0}^{1}$ of 2.2 with $\|v\|_{2}=1$, which is non-negative.

Proof. By Theorem 8.38 in [GT83], the eigenvalue $\lambda$ is simple and the eigenspace is spanned by a non-negative eigenfunction. Since $\|v\|_{2}=1$, we have a unique non-negative eigenfunction with $\|v\|_{2}=1$.

Define

$$
\Lambda=\Lambda_{\Omega}(\alpha, A)=\inf _{D \subset \Omega,|D|=A} \lambda(\alpha, A) .
$$

Remark 2.2. Assume $\alpha<\Lambda$. Then for the solution $(u, D)$ to the composite problem stated in the introduction, $|\{u=s\}|=0$ for all $s$. This is Theorem $1(\mathrm{c})$ in $\left[\mathrm{CGI}^{+} 00\right]$. Note that $s=0$ is not covered by the proof in [CGI ${ }^{+} 00$ but is easily ruled out by superharmonicity of $u$.

Lemma 2.3. Let $\mathcal{F}=\{x \in \Omega: u=c\}$, where $D=\{x \in \Omega: u \leq c\}$ and $(u, D)$ is the solution of our composite problem. Then $\nabla u \not \equiv 0$ on $\mathcal{F}$. (In fact, $\nabla u$ cannot be identically zero on the boundary of a connected component of ${ }^{c} D$.) 
Proof. Assume $\left.\nabla u\right|_{\mathcal{F}} \equiv 0$. Consider the open set $\mathcal{O}=\{x: u>c\}$. Since $A<|\Omega|$, by Remark 2.2. $|\mathcal{O}|>0$. Let $U$ be a connected component of $\mathcal{O}$, and let

$$
\left\{\begin{array}{l}
-\Delta w=\mu_{U} w \quad \text { in } U, \\
\left.w\right|_{\partial U}=0,
\end{array}\right.
$$

where $\mu_{U}$ is the first Dirichlet eigenvalue of $U$. We claim $\Lambda \leq \mu_{U}$. To check this extend $w$ to ${ }^{c} U$ by setting $w \equiv 0$ in ${ }^{c} U$. The extended function will still be denoted by $w$ and we may normalize it so that $\|w\|_{2}=1$. Then

$$
\Lambda \leq \int_{\Omega}|\nabla w|^{2}+\alpha \int_{D} w^{2}=\int_{\Omega}|\nabla w|^{2}=\mu_{U} .
$$

If $\Lambda=\mu_{U}$, then $u=w$ by Lemma 2.1 Since $w \equiv 0$ on $D$, and since $u$ is superharmonic, so is $w$, hence $w=u=0$, a contradiction. So, $\Lambda<\mu_{U}$. Let $v=\partial_{x_{j}} u$ for some fixed $j$.

In $U$,

$$
-\Delta u=\Lambda u,
$$

so that on differentiating [2.4], $v$ satisfies

$$
\left\{\begin{array}{l}
-\Delta v=\Lambda v \quad \text { in } U \\
\left.v\right|_{\partial U} \equiv 0, \quad v \in C^{\gamma}(\bar{U}) .
\end{array}\right.
$$

We claim $v \equiv 0$. This will imply $u \equiv c$ in $U$, which will contradict Remark 2.2. Since $\Lambda<\mu_{U}$, using the Fredholm alternative we may solve

$$
-\Delta f-\Lambda f=-\Lambda \quad \text { in } U, \quad f \in H_{0}^{1}(U) .
$$

Let $h=f^{+}=\max (f, 0)$. Clearly $h \in H_{0}^{1}(U)$. Multiplying 2.6 by $h$ and integrating by parts gives

$$
\int_{U}|\nabla h|^{2}-\Lambda \int_{U} h^{2}=-\int_{U} \Lambda h \leq 0
$$

Thus,

$$
\int_{U}|\nabla h|^{2} \leq \Lambda \int_{U} h^{2}
$$

If $\int_{U} h^{2} \neq 0$, then from $2.7,, \mu_{U} \leq \Lambda$. This is a contradiction. Hence $\int_{U} h^{2}=0$ and $h \equiv 0$ in $U$. Thus $f \leq 0$. Set $\psi=1-f$. Then $\psi \geq 1$, and from 2.6,

$$
-\Delta \psi-\Lambda \psi=0 .
$$

By elliptic regularity, $\psi \in C^{\infty}(U)$. Now find $U_{j} \Subset U$ with $\operatorname{dist}\left(\partial U_{j}, \partial U\right) \rightarrow 0$ and $\partial U_{j}$ smooth. So if $x \in U$, then $x \in U_{j}$ for large enough $j$. Let $\phi=v / \psi$, where $v$ is defined in 2.5. Note that, because $\psi \geq 1$, and by 2.5. again,

$$
\sup _{\partial U_{j}}|\phi| \leq \sup _{\partial U_{j}}|v| \rightarrow 0 \quad \text { as } j \rightarrow \infty .
$$


Now,

$$
\nabla \phi=\frac{\nabla v}{\psi}-\frac{v \nabla \psi}{\psi^{2}}=\frac{\psi \nabla v-v \nabla \psi}{\psi^{2}}
$$

and

$$
\begin{aligned}
\Delta \phi & =\frac{\nabla \psi \cdot \nabla v+\psi \Delta v-\nabla v \cdot \nabla \psi-v \Delta \psi}{\psi^{2}}-\frac{2}{\psi^{3}}(\psi \nabla v-v \nabla \psi) \cdot \nabla \psi \\
& =\frac{\psi \Delta v-v \Delta \psi}{\psi^{2}}-\frac{2}{\psi} \nabla \psi \cdot \nabla \phi=-\frac{2}{\psi} \nabla \psi \cdot \nabla \phi
\end{aligned}
$$

Thus $\phi$ satisfies

$$
\Delta \phi+\frac{2}{\psi} \nabla \psi \cdot \nabla \phi=0 \quad \text { in } U_{j} .
$$

Hence by the maximum principle, and 2.8,

$$
\sup _{\bar{U}_{j}}|\phi| \leq \sup _{\partial U_{j}}|\phi| \rightarrow 0 \quad \text { as } j \rightarrow \infty .
$$

Consequently, $\phi \equiv 0$ in $U$ and hence $v \equiv 0$ in $U$.

Combining Theorem 8 in [CGK00] and Lemma 2.3, we have

Lemma 2.4. If $(u, D)$ is a minimizing pair with $\alpha<\Lambda_{\Omega}$, then there exists $x_{0} \in \mathcal{F}=$ $\{u=c\}$ and $a$ ball $B\left(x_{0}, r\right)=B$ such that $B \subset \Omega$ and

$$
\mathcal{F} \cap B=\left\{(x, \phi(x)): x \in \mathbb{R}^{n-1}, \phi: U \subset \mathbb{R}^{n-1} \rightarrow \mathbb{R}\right\},
$$

with $\phi\left(x_{0}\right)=0, \nabla \phi\left(x_{0}\right)=0$ and $\phi$ real-analytic. Furthermore,

$$
\begin{aligned}
D \cap B & =\{(x, y): y<\phi(x)\} \cap B, \\
{ }^{c} D \cap B & =\{(x, y): y>\phi(x)\} \cap B .
\end{aligned}
$$

Lemma 2.5. Let $\psi=\psi\left(x^{\prime}\right): U \subset \mathbb{R}^{n-1} \rightarrow \mathbb{R}$ be smooth, where $U$ is open and $U \supset B=B(0, r)$. Assume $\psi(0)=0, \nabla \psi(0)=0$, and let

$$
D=\left\{\left(x^{\prime}, y\right): y<\psi\left(x^{\prime}\right)\right\} \cap B .
$$

Then there exists $\epsilon_{0}>0$ and a smooth function

$$
\Phi(t, x)=\Phi_{t}(x):\left\{|t| \leq \epsilon_{0}\right\} \times B \rightarrow B,
$$

where $x=\left(x^{\prime}, y\right)$, such that:

(a) For each fixed $t, \Phi_{t}: \bar{B} \rightarrow \bar{B}$ is a diffeomorphism with $\Phi(0, x)=\Phi_{0}(x)=x$.

(b) For all $t$ with $|t| \leq \epsilon_{0}$, and some $0<\delta<r / 50$,

$$
\left.\Phi_{t}\right|_{\bar{B} \backslash B(0,2 \delta)}=x .
$$


(c) Let $\chi_{D_{t}}(x)=\chi_{D}\left(\Phi_{-t}(x)\right)$. Then

$$
\left.\frac{d}{d t}\left|D_{t}\right|\right|_{t=0}=1
$$

Proof. Let $f \in C_{0}^{\infty}(B(0, \delta / 100))$ be a smooth cut-off function, $f \geq 0$. Let $v\left(x^{\prime}\right)$ denote the unit outward normal to $y=\psi\left(x^{\prime}\right)$. We extend $v\left(x^{\prime}\right)$ smoothly as a vector field $X$ to all points in $B(0, \delta / 10)$. Now define

$$
\frac{d \Phi_{t}}{d t}(x)=\frac{X(x) f(x)}{\int_{\partial D \cap B(0, \delta / 10)} f(\sigma) d \sigma}=V(x), \quad \Phi_{0}(x)=x .
$$

Then (a) and (b) follow from 2.9. Note that a simple degree argument is needed to show that $\Phi_{t}$ is a diffeomorphism. (c) follows from Appendix 1, by noting that

$$
\left.V\right|_{\partial D}=\frac{\nu\left(x^{\prime}\right) f(x)}{\int_{\partial D} f(\sigma) d \sigma} .
$$

Hence $\int_{\partial D}\langle V, v\rangle=1$.

Lemma 2.6. Construct $\Phi_{t}(x)$ as in Lemma 2.5 and suppose $x_{0}=0$ in Lemma 2.4 Define $\phi_{t}: \Omega \rightarrow \Omega$ by

$$
\phi_{t}(x)= \begin{cases}\Phi_{t}(x), & x \in B(0,3 \delta), \\ x, & x \in \Omega \backslash B(0,3 \delta) .\end{cases}
$$

(a) $\phi_{t}$ is a diffeomorphism of $\Omega$.

(b) If $D_{t}=\left\{\phi_{t}(x): x \in D\right\}$, then

$$
\frac{d}{d t}\left|D_{t}\right|=1
$$

(c) If $(u, D)$ is a solution to the composite problem and

$$
-\Delta u_{t}+\alpha \chi_{D_{t}} u_{t}=\lambda(t) u_{t},\left.\quad u_{t}\right|_{\partial \Omega}=0,
$$

where $D=\{x \in \Omega: u \leq c\}=D_{0}, u_{0}=u, \lambda(0)=\Lambda$, then

$$
\lambda^{\prime}(0)=\alpha c^{2} .
$$

Proof. Using (b) in (A1.10) and Lemma2.5 (c) we get (c); (b) follows from Lemma 2.5 , and (a) follows from the definition of $\phi_{t}(x)$ and Lemma 2.5 (a).

Lemma 2.7. Assume that $\Lambda_{\Omega}(\alpha, A)$ is differentiable at $A=A_{0}$. Let $(u, D)$ be a minimizer. Construct domains $D_{t}$ as in Lemma 2.6 where $B=B\left(x_{0}, r\right)$ is supplied by Lemma 2.4 Then

$$
\left.\frac{d}{d A} \Lambda(\alpha, A)\right|_{A=A_{0}}=\alpha c^{2}
$$


Proof. Let $\left|D_{t}\right|=m(t)$, with $D_{t}$ as in Lemma2.6 Let $f(t)=\Lambda(\alpha, m(t))$. Then $f$ is differentiable at $t=0$ and

$$
f^{\prime}(0)=\left.\frac{d \Lambda}{d A}(\alpha, A)\right|_{A=A_{0}} \cdot m^{\prime}(0)=\left.\frac{d \Lambda}{d A}(\alpha, A)\right|_{A=A_{0}} .
$$

Next for $t>0$, by the definition of $\Lambda$,

$$
\frac{f(t)-f(0)}{t} \leq \frac{\lambda(t)-f(0)}{t}=\frac{\lambda(t)-\lambda(0)}{t} .
$$

Letting $t \downarrow 0$, we get $f^{\prime}(0) \leq \lambda^{\prime}(0)$. Arguing similarly for $t<0$, letting $t \uparrow 0$, using the differentiability of $f$ and $\lambda$ at $t=0$ we get $f^{\prime}(0)=\lambda^{\prime}(0)=\alpha c^{2}$ by Lemma 2.6. Thus from $(2.10)$,

$$
\left.\frac{d \Lambda}{d A}(\alpha, A)\right|_{A=A_{0}}=\alpha c^{2}
$$

Proof of Theorem 1.2 $\Lambda(\alpha, A)$ is strictly increasing in $A$ and Lipschitz in $A\left[\mathrm{CGI}^{+} 00\right.$, Prop. 10]. Thus $\Lambda^{\prime}(\alpha, A)$ exists for a.e. $A$, and $\Lambda^{\prime}(\alpha, A)=\alpha c^{2}$ by Lemma 2.7. Hence if $\left(u_{1}, D_{1}\right),\left(u_{2}, D_{2}\right)$ are two configurations with $\left|D_{i}\right|=A$, where $D_{i}=\left\{x: u_{i}<c_{i}\right\}$, then $\alpha c_{1}^{2}=\alpha c_{2}^{2}$. Hence $c_{1}=c_{2}$.

We shall now show that under some conditions, the weak uniqueness conclusion of Theorem 1.2 can be turned into a uniqueness result. We will restrict our attention to domains $\Omega \subset \mathbb{R}^{2}$.

Lemma 2.8. Let $\Omega \subset \mathbb{R}^{2}$, and let

$$
\left\{\begin{array}{l}
-\Delta u+\alpha \chi_{\{u \leq c\}}(x) u=\lambda u \\
\left.u\right|_{\partial \Omega}=0, \quad\|u\|_{2}=1
\end{array}\right.
$$

Then for any $x_{0} \in \mathbb{R}^{2}$,

$$
\frac{1}{2} \int_{\partial \Omega}\left\langle x-x_{0}, v\right\rangle\left(\frac{\partial u}{\partial v}\right)^{2}=\lambda-\left.\alpha c^{2}\right|^{c} D \mid-\alpha \int_{D} u^{2},
$$

where $D=\{x: u \leq c\}$.

Proof. We use the Rellich-Pokhozhaev identity

$$
-\left\langle x-x_{0}, \nabla u\right\rangle \Delta u=-\nabla \cdot\left(\left\langle x-x_{0}, \nabla u\right\rangle \nabla u\right)+|\nabla u|^{2}+\frac{1}{2}\left(x-x_{0}\right) \cdot \nabla\left(|\nabla u|^{2}\right) .
$$

Integrating it over $\Omega$ yields

$$
\begin{aligned}
-\int_{\Omega}\left\langle x-x_{0}, \nabla u\right\rangle \Delta u & =-\int_{\partial \Omega}\left\langle x-x_{0}, v\right\rangle\left(\frac{\partial u}{\partial v}\right)^{2} d \sigma+\frac{1}{2} \int_{\partial \Omega}\left\langle x-x_{0}, v\right\rangle\left(\frac{\partial u}{\partial v}\right)^{2} d \sigma \\
& =-\frac{1}{2} \int_{\partial \Omega}\left\langle x-x_{0}, v\right\rangle\left(\frac{\partial u}{\partial v}\right)^{2} d \sigma
\end{aligned}
$$


From 2.11,

$$
-\Delta u=\lambda u-\alpha \chi_{D} u .
$$

Substituting (2.13) into the left side of 2.12) we get

$$
\int_{\Omega} \alpha \chi_{D} u\left\langle x-x_{0}, \nabla u\right\rangle-\int_{\Omega}\left\langle x-x_{0}, \nabla u\right\rangle \lambda u=\frac{1}{2} \int_{\Omega}\left\langle x-x_{0}, v\right\rangle\left(\frac{\partial u}{\partial v}\right)^{2} .
$$

Thus,

$$
\frac{1}{2} \int_{\Omega}\left\langle x-x_{0}, v\right\rangle\left(\frac{\partial u}{\partial v}\right)^{2}=-\frac{\lambda}{2} \int_{\Omega}\left\langle x-x_{0}, \nabla\left(u^{2}\right)\right\rangle+\frac{\alpha}{2} \int_{\Omega}\left\langle x-x_{0}, \nabla\left(u^{2}\right)\right\rangle \chi_{D} .
$$

The first integral on the right by integration by parts is

$$
\lambda \int_{\Omega} u^{2}=\lambda
$$

For the second integral, since $D=\{x: u(x) \leq c\}$, by Sard's theorem there exist $c_{j} \uparrow c$ such that each $c_{j}$ is a regular value. Let $D_{j}=\left\{x: u(x)<c_{j}\right\}$. Now by integration by parts,

$$
\begin{aligned}
\int_{\Omega}\left\langle x-x_{0}, \nabla\left(u^{2}\right)\right\rangle \chi_{D_{j}} & =-2 \int_{D_{j}} u^{2}+\int_{\partial D_{j} \cap \Omega}\left\langle x-x_{0}, v\right\rangle u^{2} \\
& =-2 \int_{D_{j}} u^{2}+\int_{\partial D_{j} \cap \Omega} c_{j}^{2}\left\langle x-x_{0}, v\right\rangle=-2 \int_{D_{j}} u^{2}-\left.2 c_{j}^{2}\right|^{c} D_{j} \mid .
\end{aligned}
$$

Letting $j \rightarrow \infty$ gives

$$
\int_{D}\left\langle x-x_{0}, \nabla\left(u^{2}\right)\right\rangle=-2 \int_{D} u^{2}-\left.2 c^{2}\right|^{c} D \mid .
$$

Inserting 2.16 and 2.15 into 2.14) we get our result.

To obtain a true uniqueness assertion we first need a preliminary lemma which is valid in all dimensions. We shall assume that our solutions are normalized by the condition $\|u\|_{2}=1$.

Lemma 2.9. Let $\left(u_{i}, D_{i}\right), i=1,2$, be two solutions of our composite problem. Assume that $D_{1}$ is connected. Assume furthermore we have weak uniqueness, that is, $D_{i}=$ $\left\{x \in \Omega: u_{i} \leq c\right\}$ and $u_{1}-u_{2}$ vanishes at a single point $x_{0} \in D_{1}$ to infinite order. Then $u_{1} \equiv u_{2}$ in $\Omega$.

Proof. First we note $u_{1}\left(x_{0}\right)=u_{2}\left(x_{0}\right)<c$. Thus there is a ball $B$ centered at $x_{0}$ where $u_{i}(x)<c, i=1,2$. In this ball we have

$$
-\Delta u_{i}+\alpha u_{i}=\Lambda u_{i}, \quad i=1,2 .
$$


Thus, $w=u_{1}-u_{2}$ also satisfies the equation 2.17) and $w$ vanishes at $x_{0}$ to infinite order. Hence, $w$ vanishes identically in $B$. Now consider the set

$$
W=\operatorname{int}\left\{x \in D_{1}: u_{1}=u_{2}\right\}
$$

We have established that $W$ is non-empty. We shall now show that $W$ is both open and closed in the relative topology of $D_{1}$. Since $D_{1}$ is connected we then get $W=D_{1}$. Since $u_{1}=u_{2}<c$ on $D_{1}^{\circ}$ we obtain $D_{1} \subset D_{2}$. Since $\left|D_{1}\right|=\left|D_{2}\right|$ we see right away that $D_{1}=D_{2}$.

Now by definition $W$ is open. So let $z_{0} \in \bar{W}=F \cap D_{1}$ where $F$ is closed. Then $u_{1}\left(z_{0}\right)=u_{2}\left(z_{0}\right)<c$ and thus there is a ball $B$ centered at $z_{0}$ where 2.17) is satisfied. Again $w$ satisfies (2.17) and vanishes on some open set in $B$. This is because $z_{0}$ is a boundary point to $W$. So by unique continuation $w$ vanishes in $B$. Thus $z_{0} \in W$. We have checked $W$ is also closed. Since now $D_{1}=D_{2}$, applying Lemma 2.1 we obtain the conclusion of our lemma.

Remark 2.10. The same result holds if $x_{0} \in \partial \Omega$. The proof is similar, but slightly more complicated.

Theorem 2.1. Assume $\Omega \subset \mathbb{R}^{2}$ with smooth boundary. Assume that $\Omega$ is strictly convex. Let $\left(u_{i}, D_{i}\right)$ be two solutions to the composite problem with eigenvalue $\Lambda$. Assume that:

(a) $\int_{D_{1}} u_{1}^{2}=\int_{D_{2}} u_{2}^{2}$.

(b) Weak uniqueness holds: $D_{i}=\left\{x \in \Omega: u_{i}(x) \leq c\right\}$.

(c) The sets $\left\{x: u_{1}(x)<u_{2}(x)\right\}$ and $\left\{x: u_{1}(x)>u_{2}(x)\right\}$ are both connected.

Then $u_{1} \equiv u_{2}$.

Proof. Since $\Omega$ is convex, it is simply connected, and since $\alpha<\Lambda$, by Theorem 2 of $\left[\mathrm{CGI}^{+} 00\right.$ the sets $D_{i}$ are connected. Writing Lemma 2.8 for $u_{i}$ and subtracting the expression for $u_{2}$ from that of $u_{1}$, we get, after using the hypotheses (a), (b) above,

$$
\int_{\partial \Omega}\left\langle x-x_{0}, v\right\rangle\left[\left(\frac{\partial u_{1}}{\partial v}\right)^{2}-\left(\frac{\partial u_{2}}{\partial v}\right)^{2}\right]=0 .
$$

We rewrite this as

$$
\int_{\partial \Omega}\left\langle x-x_{0}, v\right\rangle \frac{\partial}{\partial v}\left(u_{1}+u_{2}\right) \frac{\partial}{\partial v}\left(u_{1}-u_{2}\right)=0 .
$$

Now in a tubular neighborhood of $\partial \Omega$ both $u_{1}, u_{2}$ satisfy 2.17). Hence $u_{1}+u_{2}$ also satisfies 2.17) with $u_{1}+u_{2}>0$ in $\Omega$ and vanishing on $\partial \Omega$. Thus by Hopf's boundary point lemma,

$$
\frac{\partial}{\partial v}\left(u_{1}+u_{2}\right)<0
$$

Now set $\psi=u_{1}-u_{2}$. Let

$$
E_{1}=\left\{x \in \partial \Omega: \frac{\partial \psi}{\partial v}>0\right\}, \quad E_{2}=\left\{x \in \partial \Omega: \frac{\partial \psi}{\partial v}<0\right\} .
$$


We show both sets are empty. If we establish this result we have the conclusion of the lemma. The reason is that if $\partial \psi / \partial \nu=0$ on $\partial \Omega$, since $\psi=0$ on $\partial \Omega$ we deduce from the Cauchy-Kovalevskaya theorem that $\psi$ vanishes in a neighborhood of a boundary point, and thus applying Lemma 2.9 we conclude $u_{1}=u_{2}$ in $\Omega$.

Case 1: Assume without loss of generality that $E_{2}$ is empty and $E_{1}$ is non-empty. Pick any $x_{0} \in \Omega$. Then by the strict convexity of $\partial \Omega,\left\langle x-x_{0}, v\right\rangle>0$. Thus by (2.19) and the choice of $x_{0}$ we conclude that the integral in 2.18 is negative. This contradicts the identity 2.18.

Case 2: We may now assume that both $E_{1}$ and $E_{2}$ are non-empty. Consider the components of $E_{1}$ and $E_{2}$ on $\partial \Omega$. These are intervals. We claim that the hypothesis (c) rules out interlacing of intervals. That is, the intervals that make up the components of $E_{1}$ must share at least one boundary point, and likewise for $E_{2}$. For assume there exist two intervals $I_{1}, I_{2}$ which are components of $E_{1}$ and two intervals $J_{1}, J_{2}$ which are components of $E_{2}$. Now we shall obtain a contradiction if we assume that $I_{1}, I_{2}$ lie in different components of $\partial \Omega \backslash\left(J_{1} \cup J_{2}\right)$. Taking interior points in $I_{1}, I_{2}$ we can connect them by a curve that lies entirely in $\Omega$ and in the set $\left\{u_{1}<u_{2}\right\}$. Now it is easily seen that $\left\{u_{1}>u_{2}\right\}$ is disconnected. This contradicts (c). Thus we have shown that $\partial \Omega$ consists of two arcs $\gamma_{1}, \gamma_{2}$ such that $\gamma_{1}$ and $\gamma_{2}$ have common endpoints $P, Q$ and $\partial \psi / \partial v \geq 0$ on $\gamma_{1}$, with $\partial \psi / \partial v>0$ on some subinterval of $\gamma_{1}$. Likewise, $\partial \psi / \partial v \leq 0$ on $\gamma_{2}$, with $\partial \psi / \partial v<0$ on some subinterval of $\gamma_{2}$. Now consider the tangent lines to $\bar{\partial} \Omega$ at $P, Q$.

If the tangent lines intersect at $x_{0}$, apply 2.18 with this choice of $x_{0}$. Notice that by the strict convexity of $\partial \Omega,\left\langle x-x_{0}, v\right\rangle>0$ (except possibly at $P, Q$ ) on $\gamma_{1}$ and $\left\langle x-x_{0}, \nu\right\rangle<0$ on $\gamma_{2}$. Thus using (2.19) and the behavior of $\psi$ on $\gamma_{1}, \gamma_{2}$ we easily see that the integral in 2.18) is negative. This is a contradiction.

Assume next that the tangent lines at $P, Q$ are parallel and (with no loss of generality) parallel to the $x_{1}$-axis, $x=\left(x_{1}, x_{2}\right)$. Set $v(x)=\left(n_{1}(x), n_{2}(x)\right)$. Now 2.18) holds for every $x_{0}$. Set $x_{0}=\left(x_{1}^{0}, x_{2}^{0}\right)$. We may now differentiate 2.18 with respect to $x_{1}^{0}$ to obtain

$$
\int_{\partial \Omega} n_{1}(x) \frac{\partial}{\partial v}\left(u_{1}+u_{2}\right) \frac{\partial \psi}{\partial v}=0 .
$$

We may assume that $n_{1}(x)>0$ on $\gamma_{1}$ and $n_{1}(x)<0$ on $\gamma_{2}$ except at $P, Q$ by the strict convexity of $\partial \Omega$. Thus the integrand in 2.18 is non-positive by the use of 2.19 . Furthermore, from 2.19 and the behavior of $\psi$ on the arcs $\gamma_{i}$ there are arcs on $\partial \Omega$ where the integrand is negative. This again contradicts 2.18 . Thus both sets $E_{1}$ and $E_{2}$ are empty.

\section{Partial regularity}

Our goal in this section is to prove Theorems 1.4 and 1.5 of the introduction. We follow the works of Blank [Bla04], Shahgolian [Sha], Weiss [Wei98] and Monneau-Weiss [MW07], with some necessary variants and extensions. 
The set-up. Let $\Omega \subset \mathbb{R}^{n}$ be a bounded domain with $\partial \Omega$ smooth. For $\alpha>0$ and $A \in(0,|\Omega|)$, we let $(u, D)$ be a solution of the composite problem, so that

$$
\left\{\begin{array}{l}
-\Delta u+\alpha \chi_{\{u \leq c\}} u=\Lambda u \quad \text { on } \Omega \\
\left.u\right|_{\partial \Omega}=0, \quad \int_{\Omega} u^{2}=1
\end{array}\right.
$$

where $D=\{u \leq c\}$. Recall that $u \geq 0$ in $\Omega$ and that we are assuming throughout that $\alpha<\Lambda$. Note that $u \in W^{2, p}(\Omega)$ for all $1 \leq p<\infty$, and $u \in C^{1, \gamma}(\bar{\Omega}), 0 \leq \gamma<1$, with norm depending only on $A, n, \Omega, p, \gamma, \alpha$, and $\Lambda$. Note also that $c>0$ since if $u\left(x_{0}\right)=0$ by superharmonicity of $u, x_{0} \in \partial \Omega$, and $|\{u \leq c\}|=A>0$. Note also that $|\{u=c\}|=0$ by Remark 2.2. We next let $v=c-u$ and write the equation for $v$, namely

$$
\Delta v=f \chi\{v \geq 0\}-g \chi\{v<0\},
$$

where $f=(\Lambda-\alpha) u, g=-\Lambda u$. Fix a neighborhood $U$ of $\mathcal{F}=\{u=c\}$, the free boundary, so that $f>0, g<0$ and $f+g<0$ in $\bar{U}$. We thus have a solution $v$ of $\sqrt{3.2}$ in $U$ open, and functions $f, g \in C^{1, \gamma}(\bar{U})$ with norm bounded by $\tilde{B}_{1}=\tilde{B}_{1}(\gamma, u, \alpha, \Lambda, A, \Omega)$ in $\bar{U}$, also satisfying $f, g \in W^{2, p}(U)$, with norm bounded by $\tilde{B}_{2}=\tilde{B}_{2}(p, n, \alpha, \Lambda, A, \Omega)$ and with $|\Delta f|,|\Delta g|$ bounded by $\tilde{B}_{3}=\tilde{B}_{3}(\alpha, \Lambda)$, and such that, for some $\eta_{0}=\eta_{0}(\alpha, \Lambda, A, n, \Omega)>0$, we have $f \geq \eta_{0}>0, g \leq-\eta_{0}, f+g \leq \eta_{0}$ in $\bar{U}$. We also have $\|v\|_{C^{1, \gamma}(\bar{U})}+\|v\|_{W^{2, p}(\bar{U})} \leq N=N(\gamma, p, n, x, \Lambda, A, \Omega)$. Finally, we fix $r_{0}$ so small that $B\left(x_{0}, r_{0}\right) \subset U$ for all $x_{0} \in \mathcal{F}$. We still study the behavior of $S_{u}=\{x \in \mathcal{F}: \nabla u(x)=0\}=S_{v}=\{x \in \mathcal{F}: \nabla v(x)=0\}$, where $\mathcal{F}=\{v=0\}$. Note that by [CGK00, Theorem 8] (see Theorem 1.3 here) for each $x_{0} \in \mathcal{F} \backslash S_{v}$, there exists a neighborhood $V_{x_{0}}$ around $x_{0}$ so that $\mathcal{F}$ is real-analytic in it, and $v$ and $u$ are real-analytic in $V_{x_{0}} \cap \bar{D}$ and $V_{x_{0}} \cap \overline{{ }^{c} D}$. One of our main tools in this section is an energy functional introduced by Weiss:

$$
W(r)=\frac{1}{r^{n+2}} \int_{B\left(x_{0}, r\right)}\left(|\nabla v|^{2}+2\left(f v^{+}+g v^{-}\right)\right)-\frac{2}{r^{n+3}} \int_{\partial B\left(x_{0}, r\right)} v^{2} .
$$

In the next lemma we compute $W^{\prime}(r)$ (see [Wei98], where the computation is also carried out).

Lemma 3.1. Let $x_{0} \in S_{v}$ and $0<r<r_{0}$. Then, for $0<r<r_{0}$,

$$
W^{\prime}(r)=\frac{2}{r^{n+2}} \int_{\partial B_{r}}\left[\frac{\partial v}{\partial v}-2 \frac{v}{r}\right]^{2} d \sigma+e(r),
$$

where for $0 \leq \gamma<1$ and $0<r<r_{0}$ we have

$$
|e(r)| \leq F\left(n, \gamma,\|\nabla f\|_{\infty},\|\nabla g\|_{\infty}, N\right) r^{\gamma-1},
$$

with $F(-,-, 0,0,-) \equiv 0$. (Here $v$ is the outward unit normal to $\partial B_{r}$, and $B_{r}$ stands for $B\left(x_{0}, r\right)$.) 
Proof. We can assume that $x_{0}=0$. We have

$$
\frac{\partial}{\partial r}\left(\frac{1}{r^{n+2}} \int_{B_{r}}|\nabla v|^{2}\right)=-\frac{n-2}{r^{n+3}} \int_{B_{r}}|\nabla v|^{2}+\frac{1}{r^{n+2}} \int_{\partial B_{r}}|\nabla v|^{2} .
$$

Moreover, the Rellich-Pokhozhaev identity gives

$$
\operatorname{div}\left(x|\nabla v|^{2}\right)=2 \operatorname{div}(x \cdot \nabla v \nabla v)+(n-2)|\nabla v|^{2}-2 x \cdot \nabla v \Delta v,
$$

and we also have the identities

$$
\begin{gathered}
\left(f \chi_{\{v \geq 0\}}-g \chi_{\{v<0\}}\right) \nabla v=\nabla\left(f v^{+}+g v^{-}\right)-\nabla f v^{+}-\nabla g v^{-}, \\
\int_{B_{r}} x \cdot \nabla\left(f v^{+}+g v^{-}\right)=r \int_{\partial B_{r}}\left(f v^{+}+g v^{-}\right)-n \int_{B_{r}}\left(f v^{+}+g v^{-}\right),
\end{gathered}
$$

so that

$$
\begin{aligned}
\int_{\partial B_{r}}|\nabla v|^{2}= & 2 \int_{\partial B_{r}}\left(\frac{\partial v}{\partial v}\right)^{2}+\frac{n-2}{r} \int_{\partial B_{r}}|\nabla v|^{2}-2 \int_{B_{r}}\left(f v^{+}+g v^{-}\right) \\
& +\frac{2 n}{r} \int_{B_{r}}\left(f v^{+}+g v^{-}\right)+\frac{2}{r} \int_{B_{r}}\left[(x \cdot \nabla f) v^{+}+(x \cdot \nabla g) v^{-}\right]
\end{aligned}
$$

and hence

$$
\begin{aligned}
\frac{\partial}{\partial r}\left(\frac{1}{r^{n+2}} \int_{B_{r}}|\nabla v|^{2}\right)= & -\frac{4}{r^{n+3}} \int_{\partial B_{r}} v \frac{\partial v}{\partial v}+\frac{2(n+2)}{r^{n+3}} \int_{B_{r}}\left(f v^{+}+g v^{-}\right) \\
& +\frac{2}{r^{n+2}} \int_{\partial B_{r}}\left(\frac{\partial v}{\partial v}\right)^{2}-\frac{2}{r^{n+2}} \int_{\partial B_{r}}\left(f v^{+}+g v^{-}\right) \\
& +\frac{2}{r^{n+3}} \int_{B_{r}}\left[(x \cdot \nabla f) v^{+}+(x \cdot \nabla g) v^{-}\right],
\end{aligned}
$$

where we have also used the identity

$$
\begin{aligned}
-\frac{4}{r^{n+3}} \int_{B_{r}}|\nabla v|^{2} & =-\frac{2}{r^{n+3}} \int_{B_{r}}\left[\Delta\left(v^{2}\right)-2 v \Delta v\right] \\
& =-\frac{4}{r^{n+3}} \int_{\partial B_{r}} v \frac{\partial v}{\partial v}+\frac{4}{r^{n+3}} \int_{\partial B_{r}}\left(f v^{+}+g v^{-}\right) .
\end{aligned}
$$

Since

$$
\begin{aligned}
\frac{\partial}{\partial r}\left(\frac { 2 } { r ^ { n + 2 } } \int _ { B _ { r } } \left(f v^{+}\right.\right. & \left.\left.+g v^{-}\right)\right) \\
& =\frac{-2(n+2)}{r^{n+3}} \int_{B_{r}}\left(f v^{+}+g v^{-}\right)+\frac{2}{r^{n+3}} \int_{\partial B_{r}}\left(f v^{+}+g v^{-}\right) d \sigma
\end{aligned}
$$

and

$$
\frac{\partial}{\partial r}\left(\frac{2}{r^{n+3}} \int_{\partial B_{r}} v^{2}\right)=-\frac{8}{r^{n+3}} \int_{\partial B_{r}} v^{2}+\frac{4}{r^{n+3}} \int_{\partial B_{r}} v \frac{\partial v}{\partial v},
$$


(3.4) follows with

$$
e(r)=\frac{2}{r^{n+3}} \int_{B_{r}}\left[(x \cdot \nabla f) v^{+}+(x \cdot \nabla g) v^{-}\right] .
$$

The estimate (3.5) is an immediate consequence of this formula and the fact that $x_{0} \in S_{v}$ and $v \in C^{1, \gamma}$.

Corollary 3.2. If $f=f_{0}$ and $g=g_{0}$ are both constants, and $W^{\prime}(r)=0$ for $0<r<r_{0}$ then $v\left(x_{0}+x\right)$ is homogeneous of degree 2 in $x$.

Proof. From the formula for $W^{\prime}$ and the fact that $e \equiv 0$ in this case.

Corollary 3.3. $W_{1}(r)=W(r)+D r^{\gamma}$ (where $D=D\left(n, \gamma,\|\nabla f\|_{\infty},\|\nabla g\|_{\infty}, N\right) \geq 0$, $D(-,-, 0,0,-) \equiv 0)$ is increasing for $0<r<r_{0}$.

For further use we will recall Kato's inequality:

Lemma 3.4 (Kato [Kat73]). Assume that $w \in W_{\text {loc }}^{2,2}(U)$. Then $\Delta|w| \geq(\operatorname{sign} w) \Delta w$ in the $H_{\mathrm{loc}}^{1}(U)$ sense, i.e. for all $\theta \in C_{0}^{\infty}(U), \theta \geq 0$, we have

$$
-\int \nabla|w| \cdot \nabla \theta \geq \int(\operatorname{sign} w) \Delta w \theta .
$$

Lemma 3.5. For $0<r<r_{0}$ and $x_{0} \in S_{v}$, we have

$$
\frac{\partial}{\partial r}\left(\frac{1}{2 r^{n+3}} \int_{\partial B_{r}} v^{2}\right)=\frac{1}{r}\left[W_{1}(r)-\frac{1}{r^{n+2}} \int_{B_{r}}\left[f v^{+}+g v^{-}\right]-D r^{\gamma}\right] .
$$

Proof. Recall from the proof of Lemma 3.1 that

$$
\frac{\partial}{\partial r}\left(\frac{1}{2 r^{n+3}} \int_{\partial B_{r}} v^{2}\right)=-\frac{2}{r^{n+4}} \int_{\partial B_{r}} v^{2}+\frac{1}{r^{n+3}} \int_{\partial B_{r}} v \frac{\partial v}{\partial v} .
$$

But

$$
\begin{aligned}
\int_{\partial B_{r}} v \frac{\partial v}{\partial v} & =\frac{1}{2} \int_{\partial B_{r}} \frac{\partial}{\partial r}\left(v^{2}\right)=\frac{1}{2} \int_{B_{r}} \Delta\left(v^{2}\right) \\
& =\int_{B_{r}}\left[v \Delta v+|\nabla v|^{2}\right]=\int_{B_{r}}|\nabla v|^{2}+\int_{B_{r}}\left[f v^{+}+g v^{-}\right]
\end{aligned}
$$

and the lemma follows.

We now let, for $0<r<r_{0}$,

$$
v_{r}(x)=\frac{v\left(r x+x_{0}\right)}{r^{2}}, \quad f_{r}(x)=f\left(r x+x_{0}\right), \quad g_{r}(x)=g\left(r x+x_{0}\right),
$$

where $x_{0} \in S_{v}$. Note that $\Delta v_{r}=f_{r} \chi_{\left\{v_{r} \geq 0\right\}}-g_{r} \chi_{\left\{v_{r}<0\right\}}$ in $B_{1}=B(0, r)\left(x_{0}=0\right)$. 
Lemma 3.6. Let

$v_{r}^{(1)}(x)=f_{r}(x) v_{r}^{+}(x)+\left(g_{r}(x)+\eta_{0} / 2\right) v^{-}(x)-a_{1}|x|^{2}, \quad v_{r}^{(2)}(x)=v_{r}^{-}(x)+a_{2}|x|^{2}$, where $a_{i}=a_{i}\left(n, \tilde{B}_{1}, \tilde{B}_{2}, \tilde{B}_{3}, N, \alpha, \Lambda\right) \geq 0$. Then:

(i) $v_{r}^{(1)}$ is superharmonic in $B_{1}$.

(ii) $v_{r}^{(2)}$ is subharmonic and non-negative in $B_{1}$.

(iii) $v_{r}^{+}$is subharmonic in $B_{1}$.

Proof. All functions are continuous, so we just need to check the sign of the distributional Laplacian. Note that

$$
\begin{aligned}
v_{r}^{(1)}(x)= & \frac{f_{r}(x)+g_{r}(x)+\eta_{0} / 2}{2}\left|v_{r}(x)\right|+\frac{f_{r}(x)-g_{r}(x)-\eta_{0} / 2}{2} v_{r}(x)-a_{1}|x|^{2}, \\
\Delta v_{r}^{(1)}= & \frac{f_{r}+g_{r}+\eta_{0} / 2}{2} \Delta\left(\left|v_{r}\right|\right)+\frac{f_{r}-g_{r}-\eta_{0} / 2}{2} \Delta v_{r}(x) \\
& +2 \frac{\nabla\left(f_{r}+g_{r}\right)}{2} \nabla\left(\left|v_{r}\right|\right)+2 \frac{\Delta\left(f_{r}-g_{r}\right)}{2} v_{r} \\
& +\frac{\Delta\left(f_{r}+g_{r}\right)}{2}\left|v_{r}\right|+\frac{\Delta\left(f_{r}-g_{r}\right)}{r} v_{r}-a_{1} 2 n \\
\leq & \frac{f_{r}+g_{r}+\eta_{0} / 2}{2}\left(\operatorname{sign} v_{r}\right)\left(f_{r} \chi_{\left\{v_{r} \geq 0\right\}}-g_{r} \chi_{\left\{v_{r}<0\right\}}\right) \\
& +\frac{f_{r}-g_{r}-\eta_{0} / 2}{2}\left(\operatorname{sign} v_{r}\right)\left(f_{r} \chi_{\left\{v_{r} \geq 0\right\}}-g_{r} \chi_{\left\{v_{r}<0\right\}}\right)+2 \tilde{B}_{2} N+2 \tilde{B}_{2} N-a_{1} 2 n \\
\leq & \tilde{B}_{2}^{2} \tilde{B}_{2}^{2}+2 \tilde{B}_{3} N-a_{1} 2 n
\end{aligned}
$$

and (i) follows. (Here we have used the fact that $f_{r}+g_{r}+\eta_{0} / 2<0$.) Also,

$$
v_{r}^{(2)}(x)=\frac{\left|v_{r}(x)\right|-v_{r}(x)}{2}+a_{2}|x|^{2},
$$

so that

$$
\begin{aligned}
\Delta v_{r}^{(2)} & =\frac{\left(\operatorname{sign} v_{r}\right) v_{r}-\Delta v_{r}}{2}+2 n a_{2} \\
& =\frac{\left(f_{r} \chi_{\left\{v_{r} \geq 0\right\}}-g_{r} \chi_{\left\{v_{r}<0\right\}}\right)-\left(f_{r} \chi_{\left\{v_{r} \geq 0\right\}}-g_{r} \chi_{\left\{v_{r}<0\right\}}\right)}{2}+2 n a_{2} \\
& \geq g_{r} \chi_{\left\{v_{r}<0\right\}}+2 n a_{2}
\end{aligned}
$$

and (ii) follows. For (iii) note that $v_{r}^{+}=\left|v_{r}^{+}\right|+v_{r}^{+} / 2$, so that

$$
\begin{aligned}
\Delta v_{r}^{+} & \geq \frac{\left(\operatorname{sign} v_{r}\right) \Delta v_{r}+\Delta v_{r}}{2}+2 n a_{2} \\
& =\frac{\left(f_{r} \chi_{\left\{v_{r} \geq 0\right\}}+g_{r} \chi_{\left\{v_{r}<0\right\}}\right)+\left(f_{r} \chi_{\left\{v_{r} \geq 0\right\}}-g_{r} \chi_{\left\{v_{r}<0\right\}}\right)}{2}=f_{r} \chi_{\left\{v_{r} \geq 0\right\}} \geq 0 .
\end{aligned}
$$


Corollary 3.7. $-\int_{B_{1}}\left[f_{r} v_{r}^{+}+\left(g_{r}+\eta_{0} / 2\right) v_{r}^{-}\right] \geq-a_{3}$, where $a_{3}>0$ has the same dependence as $a_{i}$ in Lemma 3.6 .

Proof. $v_{r}^{(1)}$ is superharmonic in $B_{1}$ and $v_{r}^{(1)}(0)=0$. Then

$$
v_{r}^{(1)}(0) \geq f_{B_{1}}\left[f_{r} v_{r}^{+}+\left(g_{r}+\eta_{0} / 2\right) v_{r}^{-}-a_{1}|x|^{2}\right]
$$

and the corollary follows.

We now define, for $x_{0} \in S_{v}, 0<r<r_{0}$,

$$
S(r)=\left(f_{\partial B_{r}} v^{2}\right)^{1 / 2} .
$$

Lemma 3.8 (Non-degeneracy). $\liminf _{r \rightarrow 0} S(r) / r^{2}>0$.

Proof. Assume without loss of generality that $x_{0}=0$. If the conclusion fails, we can find $r_{i} \rightarrow 0$ such that $S\left(r_{i}\right) / r_{i}^{2} \rightarrow 0$. Let $v_{i}(x)=v\left(r_{i} x\right) / r_{i}^{2}$, so that $\int_{\partial B_{1}} v_{i}^{2} \rightarrow 0$. Note that, in $B_{1}, \Delta v_{i}=f_{r_{i}} \chi_{\left\{v_{i} \geq 0\right\}}-g_{r_{i}} \chi_{\left\{v_{i}<0\right\}} \geq \eta_{0}>0$. Also, $\left|\Delta v_{i}\right| \leq 2 \tilde{B}_{1}$ and $v_{i}(0)=0$. By subharmonicity of $v_{i}$, we see that $\int_{B_{1}} v_{i}^{-} \leq \int_{B_{1}} v_{i}^{+}$. Since, by Lemma 3.6 (iii), $v_{i}^{+}$is subharmonic,

$$
\int_{B_{1}} v_{i}^{+} \leq c_{n} \int_{\partial B_{1}} v_{i}^{+} \leq c_{n}\left(\int_{\partial B_{1}}\left(v_{i}^{+}\right)^{2}\right)^{1 / 2} .
$$

Thus,

$$
\int_{B_{1}}\left|v_{i}\right| \leq \int_{B_{1}} v_{i}^{+}+\int_{B_{1}} v_{i}^{-} \leq 2 \int_{B_{1}} v_{i}^{+} \leq 2 c_{n}\left(\int_{\partial B_{1}}\left(v_{i}^{+}\right)^{2}\right)^{1 / 2} \rightarrow 0 .
$$

After passing to a subsequence, we have $v_{i} \rightarrow v_{0}$, where the convergence is uniform on compact subsets of $B_{1}$ and in $W_{\mathrm{loc}}^{2,2}\left(B_{1}\right)$. But then $\Delta v_{0} \geq \eta_{0}>0$, while $\int_{B_{1}}\left|v_{0}\right|=0$, a contradiction.

Remark 3.9. Note that the above proof shows that if $S^{+}(r)=\left(f_{\partial B_{r}}\left(v^{+}\right)^{2}\right)^{1 / 2}$, then $\liminf _{r \rightarrow 0} S^{+}(r) / r^{2}>0$.

We now turn to the classification of blow-up points, following the ideas of MonneauWeiss [MW07].

Lemma 3.10. Let $-M=\lim _{r \downarrow 0} W_{1}(r)$. Assume that $x_{0} \in S_{v}$ is such that $M<\infty$. Then there exists $G=G\left(n, \tilde{B}_{1}, \tilde{B}_{2}, \tilde{B}_{3}, N, M\right)$ such that $\sup _{0<r<r_{0}} S(r) / r^{2} \leq G$. 
Proof. Note that, in view of Lemma 3.5 if $0<r<r_{0}$ is such that

$$
-\frac{1}{r^{n+2}} \int_{B_{r}}\left[f v^{+}+g v^{-}\right]>M+D r^{\gamma}
$$

then

$$
\frac{\partial}{\partial r}\left(\frac{1}{r^{n+2}} \int_{\partial B_{r}} v^{2}\right)>0 .
$$

Note that the last inequality is equivalent to $(\partial / \partial r)\left(\int_{B_{1}} v_{r}^{2}\right)>0$. Our first step in the proof is to show that there exists $C_{1}=C_{1}\left(n, \tilde{B}_{1}, \tilde{B}_{2}, \tilde{B}_{3}, N, \Omega\right)$ such that for $0<r<r_{0}$ we have

$$
\int_{\partial B_{1}}\left(v_{r}^{+}\right)^{2} \leq C_{1}\left\{1+\int_{\partial B_{1}}\left(v_{r}^{-}\right)^{2}\right\} .
$$

In order to establish (3.7), we first prove an auxiliary claim:

Claim 3.11. For each $R>0$, there exists $\epsilon_{0}=\epsilon_{0}(R, n)$ such that if $w(0)=0, \Delta w^{+} \geq 0$, $0 \leq \Delta w \leq \epsilon_{0}, \int_{B_{1}}|\nabla w|^{2} \leq R, \int_{\partial B_{1}}\left(w^{-}\right)^{2} \leq \epsilon_{0}$ and $\left(\int_{\partial B_{1}} w^{2}\right)^{1 / 2} \leq 2$, then $\int_{\partial B_{1}} w^{2}$ $\leq 1 / 2$.

Proof of Claim 3.11. If not, we can find $R>0$ and functions $w_{j}$ with $w_{j}(0)=0,0 \leq$ $\Delta w_{j} \leq 1 / j, \int_{B_{1}}\left|\nabla w_{j}\right|^{2} \leq R,\left(\int_{\partial B_{1}} w_{j}^{2}\right)^{1 / 2} \leq 2, \int_{\partial B_{1}}\left(w_{j}^{-}\right)^{2} \leq 1 / j$ but $\int_{\partial B_{1}} w_{j}^{2} \geq 1 / 2$. Since the $w_{j}^{+}$are subharmonic, $\int_{B_{1}} w_{j}^{+} \leq c_{n} \int_{\partial B_{1}} w_{j}^{+} \leq 2 c_{n}$. Since the $w_{j}$ are subharmonic and $w_{j}(0)=0, \int_{B_{1}} w_{j}^{-} \leq \int_{B_{1}} w_{j}^{+} \leq 2 c_{n}$. Hence, by Poincaré's inequality, $\int_{B_{1}} w_{j}^{2} \leq\left(R+4 c_{n}\right) \alpha_{n}$. Hence, we can find a subsequence (still indexed by $j$ ) such that $w_{j} \rightarrow w$ uniformly on compact sets and $\int_{B_{1}}|\nabla w|^{2} \leq R$. Moreover, by compactness in the trace theorem, we have $\int_{\partial B_{1}} w^{2} \geq 1 / 2$. We also have $\Delta w=0, w(0)=0$, $\left(\int_{\partial B_{1}} w^{2}\right)^{1 / 2} \leq 2$ and $\int_{\partial B_{1}} w^{-}=0$. But then $w \geq 0, w(0)=0$ and $\Delta w=0$ imply $w \equiv 0$, a contradiction.

Suppose now that 3.7 fails for some fixed $C_{1}>1$, to be determined. Then there exists a sequence $\left\{r_{m}\right\}$ with $0<r_{m}<r_{0}$ so that $\int_{\partial B_{1}}\left(v_{r_{m}}^{+}\right)^{2} \geq C_{1}\left\{1+\int_{\partial B_{1}}\left(v_{r_{m}}^{-}\right)^{2}\right\}$. Using Corollary 3.3 we see that

$$
\begin{aligned}
\int_{B_{1}}\left|\nabla v_{r_{n}}\right|^{2}-2 \int_{\partial B_{1}} v_{r_{n}}^{2} & \leq W_{1}\left(r_{0}\right)+2 D r_{0}^{\gamma}-2 \int_{B_{1}}\left(f v_{r_{n}}^{+}+g v_{r_{n}}^{-}\right) \\
& \leq W_{1}\left(r_{0}\right)+2 D r_{0}^{\gamma}-2 \int_{B_{1}} g v_{r_{n}}^{-} .
\end{aligned}
$$

Consider now $w_{n}=v_{r_{n}} /\left(\int_{\partial B_{1}}\left(v_{r_{n}}^{+}\right)^{2}\right)^{1 / 2}$. Note that $w_{n}(0)=0, \Delta w_{n} \geq 0$, and by Lemma 3.6 (iii), we have $\Delta w_{n}^{+} \geq 0$. Also $\left(\int_{\partial B_{1}} w_{n}^{2}\right)^{1 / 2} \leq\left(1+1 / C_{1}^{1 / 2}\right) \leq 2, \int_{\partial B_{1}} w_{n}^{2} \geq$ $\int_{\partial B_{1}}\left(w_{n}^{+}\right)^{2}=1$, and $\left|\Delta w_{n}\right| \leq C / C_{1}^{1 / 2}$, where $C=C\left(\tilde{B}_{1}\right)$, since $\int_{\partial B_{1}}\left(v_{r_{n}}^{+}\right)^{2} \geq C_{1}$. Moreover, $\left(\int_{\partial B_{1}}\left(w_{n}^{-}\right)^{2}\right)^{1 / 2} \leq 1 / C_{1}^{1 / 2}$. 
But (3.8) shows that

$$
\begin{aligned}
\int_{B_{1}}\left|\nabla w_{n}\right|^{2} \leq & 2 \int_{\partial B_{1}} w_{n}^{2}+\frac{W_{1}\left(r_{0}\right)}{\int_{\partial B_{1}}\left(v_{r_{n}}^{+}\right)^{2}}+\frac{2 D r_{0}^{\gamma}}{\int_{\partial B_{1}}\left(v_{r_{n}}^{+}\right)^{2}} \\
& +2 c_{n} \tilde{B}_{1} \cdot\left(\int_{\partial B_{1}} v_{r_{n}}^{-}+a_{2}\right) / \int_{\partial B_{1}}\left(v_{r_{n}}^{+}\right)^{2}
\end{aligned}
$$

in view of Lemma 3.6 (ii). Finally, since $\int_{\partial B_{1}}\left(v_{r_{n}}^{+}\right)^{2} \geq C_{1} \geq 1,\left(\int_{\partial B_{1}} w_{n}^{2}\right)^{1 / 2} \leq 2$, and

$$
\int_{\partial B_{1}}\left(v_{r_{n}}^{-}\right)^{2} \leq\left(\int_{\partial B_{1}}\left(v_{r_{n}}^{-}\right)^{2}\right)^{1 / 2} \leq \frac{1}{C_{1}^{1 / 2}}\left(\int_{\partial B_{1}}\left(v_{r_{n}}^{+}\right)^{2}\right)^{1 / 2},
$$

we see that $\int_{B_{1}}\left|\nabla w_{n}\right|^{2} \leq R=R\left(\tilde{B}_{1}, \tilde{B}_{2}, \tilde{B}_{3}, n, N, r_{0}\right)$ for all $C_{1} \geq 1$. But if we now choose $C / C_{1} \leq \epsilon_{0}, 1 / C_{1}^{1 / 2} \leq \epsilon_{0}$, where $\epsilon_{0}$ is as in Claim 3.11, we reach a contradiction to Claim 3.11, establishing 3.7.

We now proceed to the completion of the proof of Lemma 3.10. For $0<r<r_{0}$ and $\tilde{r} \in(r / 2, r)$, we have $W_{1}(r)-W_{1}(\tilde{r}) \leq W_{1}\left(r_{0}\right)+M$. But, by Lemma 3.1 .

$$
\begin{aligned}
W_{1}(r)-W_{1}(\tilde{r}) & =\int_{\tilde{r}}^{r} W_{1}^{\prime}(s) d s \\
& =\int_{\tilde{r}}^{r} 2 s \int_{\partial B_{1}}\left(\partial_{s} v_{s}\right)^{2} d s+\int_{\tilde{r}}^{r} e(s) d s+\gamma D \int_{\tilde{r}}^{r} s^{\gamma-1} d s \\
& \geq \int_{\tilde{r}}^{r} 2 s \int_{\partial B_{1}}\left(\partial_{s} v_{s}\right)^{2} d s,
\end{aligned}
$$

by our choice of $D$ and the fact that

$$
\partial_{s} v_{s}=\frac{x \cdot \nabla v\left(s x+x_{0}\right)}{s^{3}}-\frac{2 v\left(s x+x_{0}\right)}{s^{3}} .
$$

The right hand side of the inequality above is greater than $r \int_{\tilde{r}}^{r} \int_{\partial B_{1}}\left(\partial_{s} v_{s}\right)^{2} d \sigma d s$, which by Cauchy-Schwarz is greater than $\int_{\partial B_{1}}\left(v_{r}-v_{\tilde{r}}\right)^{2} d \sigma$. Hence, for $0<r<r_{0}$ and $\tilde{r} \in(r / 2, r)$, we have

$$
\int_{\partial B_{1}}\left(v_{r}-v_{\tilde{r}}\right)^{2} \leq W_{1}\left(r_{0}\right)+M
$$

We next show:

Claim 3.12. There exists $\tilde{M}=\tilde{M}\left(n, \tilde{B}_{1}, \tilde{B}_{2}, \tilde{B}_{3}, N, M, r_{0}, \eta_{0}\right)$ such that if $\int_{\partial B_{1}} v_{r}^{2}>\tilde{M}$, then $\frac{\partial}{\partial r}\left(\int_{\partial B_{1}} v_{r}^{2}\right)>0$ for $0<r<r_{0}$. 
To establish the claim note that in light of the remark at the beginning of the proof of Lemma 3.10 , we only need to show that $\int_{\partial B_{1}} v_{r}^{2} \geq \tilde{M}$ implies

$$
-\int_{B_{1}}\left[f_{r} v_{r}^{+}+g_{r} v_{r}^{-}\right]>M+D r^{\gamma} .
$$

By Corollary 3.7

$$
-\int_{B_{1}}\left[f_{r} v_{r}^{+}+\left(g_{r}+\eta_{0} / 2\right) v_{r}^{-}\right] \geq-a_{3}
$$

so it is enough to show that

$$
\eta_{0} \int_{B_{1}} v_{r}^{-}>M+D r^{\gamma}+a_{3}
$$

From Lemma 3.6(ii), we have (by interior estimates)

$$
\left(\int_{1 / 2<|x|<3 / 4}\left(v_{r}^{-}+a_{2}|x|^{2}\right)^{2}\right)^{1 / 2} \leq c_{n} \int_{B_{1}}\left(v_{r}^{-}+a_{2}|x|^{2}\right)
$$

so that

$$
\int_{B_{1}} v_{r}^{-} \geq \frac{1}{c_{n}}\left(\int_{1 / 2<|x|<3 / 4}\left(v_{r}^{-}\right)^{2}\right)^{1 / 2}-\tilde{c}_{n} .
$$

But $\int_{1 / 2<|x|<3 / 4}\left(v_{r}^{-}\right)^{2} \geq a_{n} \int_{1 / 2}^{3 / 4} \int_{\partial B_{1}}\left(v_{r s}^{-}\right)^{2} d \sigma d s$ and

$$
\int_{\partial B_{1}}\left(v_{r s}^{-}\right)^{2}=\int_{\partial B_{1}}\left[v_{r s}^{2}-\left(v_{r s}^{+}\right)^{2}\right] \geq \int_{\partial B_{1}}\left(v_{r s}\right)^{2}-C_{1}-C_{1} \int_{\partial B_{1}}\left(v_{r s}^{-}\right)^{2},
$$

from 3.7. Thus,

$$
\int_{\partial B_{1}}\left(v_{r s}^{-}\right)^{2} \geq \frac{1}{1+C_{1}} \int_{\partial B_{1}} v_{r s}^{2}-\frac{C_{1}}{C_{1}+1}
$$

and so from 3.11 we obtain

$$
\int_{B_{1}} v_{r}^{-} \geq d_{n}\left(\int_{1 / 2}^{3 / 4} \int_{\partial B_{1}} v_{r s}^{2} d \sigma d s\right)-C_{2}
$$

with $C_{2}$ having the same dependence as $C_{1}$. If we now use 3.8 with $\tilde{r}=r s$, we see, using (3.9), that

$$
\int_{B_{1}} v_{r}^{-} \geq \tilde{d}_{n}\left(\int_{\partial B_{1}} v_{r}^{2}\right)^{2}-b_{n}\left(W_{1}\left(r_{0}\right)+M\right)^{1 / 2}-C_{2},
$$

and 3.10 holds for $\tilde{M}$ large enough.

We can now conclude the proof of Lemma 3.10 if $S(r) / r^{2} \leq \tilde{M}$ for $0<r<r_{0}$, we are done. If $S(r) / r^{2}>\tilde{M}$ for all $0<r<r_{0}$, then by Claim 3.12 we have $S(r) / r^{2}=$ $\int_{\partial B_{1}} v_{r}^{2}<S\left(r_{0}\right) / r_{0}^{2}$ for $0<r<r_{0}$ and we are also done. Note that if $S\left(r_{1}\right) / r_{1}^{2}>\tilde{M}$ for some $0<r_{1}<r_{0}$, then $S(r) / r^{2}>\tilde{M}$ for all $r_{1}<r<r_{0}$ by virtue of Claim 3.12 It is now easy to show that $S(r) / r^{2} \leq \max \left(\tilde{M}, S\left(r_{0}\right) / r_{0}\right)$ for all $0<r<r_{0}$. Thus, Lemma 3.10 follows. 
Corollary 3.13. Let $M$ and $G$ be as in Lemma 3.10 Then there exists $\tilde{G}$, with the same dependence as $G$, such that, for all $0<r<r_{0} / 2$,

$$
\sup _{|x| \leq 1}\left|v_{r}(x)\right|+\left(\int_{B_{1}}\left|\nabla v_{r}\right|^{2}\right)^{1 / 2} \leq \tilde{G} .
$$

Proof. By Corollary 3.3, for $0<r<r_{0}$ we have

$$
\begin{aligned}
\int_{B_{1}}\left|\nabla v_{r}\right|^{2} & \leq 2 \int_{\partial B_{1}} v_{r}^{2}-2 \int_{B_{1}}\left(f v_{r}^{+}+g v_{r}^{-}\right)+2 D r_{0}^{\gamma}+W_{1}\left(r_{0}\right) \\
& \leq 2 \int_{\partial B_{1}} v_{r}^{2}-2 \int_{B_{1}} g v_{r}^{-}+2 D r_{0}^{\gamma}+W_{1}\left(r_{0}\right) .
\end{aligned}
$$

Now Lemmas 3.6 ii) and 3.10 yield the gradient estimate. For the $L^{\infty}$ estimate, we use Lemma 3.6 (i), (ii) and the fact that for non-negative subharmonic functions, the $L^{2}$ spherical averages are increasing. Thus, for instance,

$$
\begin{aligned}
\sup _{|x| \leq 1}\left|v_{r}^{+}(x)\right| & \leq \sup _{|x|=1}\left|v_{r}^{+}(x)\right| \leq \tilde{c}_{n}\left(\int_{\partial B_{1}}\left|v_{r}^{+}\right|^{2}\right)^{1 / 2} \\
& \leq c_{n}\left(\int_{1 / 2<|x|<3 / 2}\left|v_{r}^{+}\right|^{2}\right)^{1 / 2} \leq \tilde{c}_{n}\left(\int_{\partial B_{1}}\left(v_{2 r}^{+}\right)^{2}\right)^{1 / 2},
\end{aligned}
$$

and similarly for $v_{r}^{-}$.

We are now ready, in analogy with [MW07], to state our classification of blow-up points.

Theorem 3.1. Assume that $x_{0} \in S_{v}$ and $W_{1}(r)$ is defined in Corollary 3.3

(i) If $\lim _{r \downarrow 0} W_{1}(r)=-M, M<\infty$, then $S(r) / r^{2}$ and $\left\|v_{r}\right\|_{W^{2, p}\left(B_{1}\right)}, 1<p<\infty$, remain bounded for $0<r<r_{0} / 2$. Moreover, if $\left\{r_{j}\right\}$ is a sequence tending to 0 , then after passing to a subsequence $\left\{r_{j^{\prime}}\right\}$, the functions $v_{r_{j^{\prime}}}$ converge in $C^{1, \gamma}\left(B_{1}\right)$, $0 \leq \gamma<1$, and $W^{2, p}\left(B_{1}\right), 1 \leq p<\infty$, to a function $\bar{v}$. The function $\bar{v}$ solves the equation

$$
\Delta \bar{v}=f_{0} \chi_{\{\bar{v} \geq 0\}}-g_{0} \chi_{\{\bar{v}<0\}} \quad \text { in } \mathbb{R}^{n}
$$

with $f_{0}=f\left(x_{0}\right), g_{0}=g\left(x_{0}\right)$, and is homogeneous of degree 2 .

(ii) If $\lim _{r \downarrow 0} W_{1}(r)=-\infty$, then $\lim _{r \downarrow 0} S(r) / r^{2}=+\infty$. Let $r_{j} \downarrow 0$ and define $w_{j}(x)=$ $v\left(r_{j} x+x_{0}\right) / S\left(r_{j}\right)$ and $T_{j}=S\left(r_{j}\right) / r_{j}^{2}$. Then, after passing to a subsequence $\left\{r_{j^{\prime}}\right\}$, the $w_{j^{\prime}}$ converge in $C^{1, \gamma}\left(B_{1}\right)$ and $W^{2, p}\left(B_{1}\right), 0 \leq \gamma<1,1 \leq p<\infty$, to a harmonic function $\bar{w}$ with $\bar{w}(0)=\nabla \bar{w}(0)=0$, which is non-zero and homogeneous of degree 2 .

Proof. From Corollary 3.13, in case (i) it only remains to show that $\bar{v}$ is homogeneous of degree 2. But this follows from Corollary 3.2 since for any $0<s<1$ we have $W(s)=W(s ; 0 ; \bar{v})=\lim _{j^{\prime} \rightarrow \infty} W_{1}\left(s r_{j^{\prime}}, x_{0}, v\right)=-M$. For case (ii), we must have

$$
\lim _{r \downarrow 0} \int_{B_{1}}\left|\nabla v_{r}\right|^{2}+2 \int_{B_{1}} f v_{r}^{2}+2 \int_{B_{1}} g v_{r}^{-}-2 \int_{\partial B_{1}} v_{r}^{2}=-\infty .
$$


But then, since $f>0$ and $g<0$, we must have

$$
\lim _{r \rightarrow 0} 2 \int_{\partial B_{1}} v_{r}^{2}-2 \int_{B_{1}} g v_{r}^{-}=+\infty
$$

By Lemma 3.6(ii),

$$
\int_{B_{1}} v_{r}^{-} \leq c_{n} a_{2}+c_{n} \int_{\partial B_{1}} v_{r}^{-} \leq c_{n} a_{2}+\left(\int_{\partial B_{1}}\left(v_{r}^{-}\right)^{2}\right)^{1 / 2} .
$$

Since $-g \geq \eta_{0}$ and $-g \leq \tilde{B}_{1}$, we conclude from 3.12 that $\lim _{r \rightarrow 0} \int_{\partial B_{1}} v_{r}^{2}+\int_{B_{1}} v_{r}^{-}=$ $+\infty$, which in turn implies $\lim _{r \rightarrow 0} \int_{\partial B_{1}} v_{r}^{2}=+\infty$, or $\lim _{r \rightarrow 0} S(r) / r^{2}=+\infty$. By Corollary 3.3 dividing by $T_{j}^{2}$, we obtain

$$
\int_{B_{1}}\left|\nabla w_{j}\right|^{2} \leq \frac{W_{1}\left(r_{0}\right)}{T_{j}^{2}}+\frac{2}{T_{j}} \int_{B_{1}}\left[f_{r_{j}} w_{j}^{+}+g_{r_{j}} w_{j}^{-}\right]+2 \int_{\partial B_{1}} w_{j}^{2}-\frac{D r_{j}^{\gamma}}{T_{j}^{2}} .
$$

Also, for $j$ large, $\left|\Delta w_{j}\right| \leq 1$ in $B_{1}, \int_{\partial B_{1}} w_{j}^{2}=1, \Delta w_{j}^{+} \geq 0, \Delta w_{j} \geq 0$ and $w_{j}(0)=0$. Then $\int_{B_{1}} w_{j}^{2} \leq C$ and from the formulae above, $\int_{B_{1}}\left|\nabla w_{j}\right|^{2} \leq 3$ for $j$ large. Thus, the $w_{j}$, after passing to a subsequence, converge uniformly on compacts and in $C^{1, \gamma}\left(B_{1}\right)$ and $W^{2, p}\left(B_{1}\right)$ to a $\bar{w}$ which is harmonic in $B_{1}$ with $\bar{w}(0)=\nabla \bar{w}(0)=0$. Also, by compactness of the trace operator, $\int_{\partial B_{1}} \bar{w}^{2}=1$, so that $\bar{w}$ is not zero. But, from 3.13 , we conclude that $\int_{B_{1}}|\nabla \bar{w}|^{2} \leq 2 \int_{\partial B_{1}}|\bar{w}|^{2}$. Hence by the Almgren monotonicity formula (see for example Lemma 4.2 in [MW07]), $w$ is homogeneous of degree 2.

Corollary 3.14 (No mixed asymptotics). For two sequences $\left\{r_{j}\right\},\left\{\tilde{r}_{j}\right\}$, both tending to zero, we cannot have

$$
\lim _{j \rightarrow \infty} \frac{S\left(r_{j}\right)}{r_{j}^{2}}=+\infty, \quad \text { but } \sup _{j} \frac{S\left(\tilde{r}_{j}\right)}{\tilde{r}_{j}^{2}}<+\infty .
$$

Proof. If $\lim _{r \downarrow 0} W_{1}(r)=-\infty$, then for all such sequences the limit is $+\infty$. On the other hand, if $\lim _{r \downarrow 0} W_{1}(r)>-\infty$, we have boundedness near $r=0$. In either case the mixed asymptotic assumption leads to a contradiction.

We will next use these results to study partial regularity of the free boundary $\mathcal{F}$. We start with a 2-dimensional result, due to Shahgholian [Sha].

Theorem 3.2 ([Sha] $)$. Let $v$ be the solution of (3.2), when $n=2$, under our assumptions. Assume that $x_{0} \in S_{v}$ is such that $\left|\{v<0\} \cap B\left(x_{0}, r\right)\right| \geq c_{0} r^{2}$ for $0<r<r\left(x_{0}\right)$, with $c_{0}>0$. Then $x_{0}$ is an isolated point of $S_{v}$.

We will provide a proof of this theorem (following [Sha $]$ ) for the reader's convenience. The key point is the following 
Lemma 3.15 ([Sha] $)$. Assume that $\bar{v}$ is a homogeneous (of degree 2) solution to (3.2) in $\mathbb{R}^{2}$, with $f=f_{0}, g=g_{0}$, both constants. (As before, $f_{0}>0, g_{0}<0, f_{0}+g_{0}<0$.) Then $S_{\bar{v}}=\{0\}$, or, after rotation, $S_{\bar{v}}=\left\{\left(x_{1}, x_{2}\right)=\left(0, x_{2}\right): x_{2} \in \mathbb{R}\right\}$. In this case $\bar{v}=\left(f_{0} / 2\right) x_{1}^{2}$.

Proof. Recall that $\Delta \bar{v} \geq \eta_{0}>0\left(\eta_{0}=\min \left(f_{0}, g_{0}\right)\right)$. Assume that $S_{v} \neq\{0\}$. After rotation we can assume that, by the homogeneity of $\bar{v},(0,1) \in S_{\bar{v}}$, so that $\lambda(0,1) \in S_{\bar{v}}$, $\lambda>0$. Assume first that $\bar{v} \geq 0$ in a neighborhood of $(0,1)$. Then, in an angle, $\Delta \bar{v}=f_{0}$. Consider $w=\bar{v}-\left(f_{0} / 2\right) x_{1}^{2}$. Then, in this angle, by uniqueness for the Cauchy problem, $w \equiv 0$. But this argument can be continued all around, so that $\bar{v}=\left(f_{0} / 2\right) x_{1}^{2}$. Thus, if not, there exists a neighborhood of $(0,1)$ in which $\bar{v}<0$ is non-empty. Assume, for instance, that the negative point is in the top right quadrant. By homogeneity, the point can be taken on the unit circle. But then all the points on the unit circle between this point and the vertical axis are points where $\bar{v}$ is negative, as otherwise we would have a local maximum, contradicting the subharmonicity of $\bar{v}$. Then, if we consider a small half-ball in the top right quadrant, centered at $(0,1)$, the Hopf maximum principle yields a contradiction to $\bar{v}(0,1)=0, \nabla \bar{v}(0,1)=0$.

Proof of Theorem 3.2. We can assume that $x_{0}=0$. Suppose we have $x_{j} \in S_{v}$ and $x_{j} \rightarrow 0$. Let $r_{j}=\left|x_{j}\right|$. Assume first that $\lim _{r \downarrow 0} W_{1}(r)=-\infty$. Then, by Theorem 3.1 (ii), $v\left(r_{j} x\right) / S\left(r_{j}\right)$, after passing to a subsequence, converges in $C^{1, \gamma}\left(B_{1}\right)$ and in $L^{2}\left(\partial B_{1}\right)$ to a harmonic polynomial $\bar{w}$ homogeneous of degree 2 and non-zero. Moreover, $x_{j} /\left|x_{j}\right| \rightarrow$ $\bar{x} \in \partial B_{1}$, and $\bar{w}(\bar{x})=0, \nabla \bar{w}(\bar{x})=0$. But, when $n=2, \bar{w}$ must be a rotate of $a\left(x_{1}^{2}-x_{2}^{2}\right)$ and hence $S_{\bar{w}}=\{0\}$, a contradiction. If $\lim _{r \downarrow 0} W_{1}(r)>-\infty$, by Theorem 3.1(i), $v\left(r_{j} x\right) / r_{j}^{2}$ converges, after passing to a subsequence, to a $\bar{v}$, a homogeneous solution of degree 2 , for $f=f_{0}, g=g_{0}$. Clearly $\left|\{\bar{v}<0\} \cap B_{1}\right| \geq c_{0}$. Also, $\bar{x} \in S_{\bar{v}}$, so that by Lemma $3.15, \bar{v}=\left(f_{0} / 2\right) x_{1}^{2}$, after a rotation, which is a contradiction.

We will next extend Theorem 3.2 to $n>2$. The argument is standard in the theory of minimal surfaces (see Chapter 11 of [Giu84], whose notation for Hausdorff measures and Hausdorff dimension we adopt). Similar arguments have been used by Weiss [Wei98] and Monneau-Weiss [MW07] in the context of free boundary problems. Our result here is:

Theorem 3.3. Let $v$ be a solution of 3.2$\}, n \geq 2$, under our assumptions. Define $\tilde{S}_{v}=$ $\left\{x_{0} \in S_{v}:\left|\{v<0\} \cap B\left(x_{0}, r\right)\right| \geq c_{0} r^{n}\right.$ for $\left.0<r<r_{0}\left(x_{0}\right)\right\}$. Then, for each fixed $c_{0}>0$, the Hausdorff dimension of $\tilde{S}_{v}$ is at most $n-2$.

Proof. Fix $k>n-2$. We need to show that $H_{k}\left(\tilde{S}_{v}\right)=0$. Assume not, so that $H_{k}\left(\tilde{S}_{v}\right)>0$. Consider the sets

$$
\tilde{S}_{v}^{j}=\left\{x_{0} \in S_{v}:\left|\{v \leq 0\} \cap B\left(x_{0}, r\right)\right| \geq c_{0} r^{n} \text { for } 0<r<1 / j\right\} .
$$

Then $\tilde{S}_{v}=\bigcup_{j=j_{0}}^{\infty} \tilde{S}_{v}^{j}$, where $1 / j_{0}<r_{0}$. Hence $H_{k}\left(\tilde{S}_{v}^{\bar{j}}\right)>0$ for some $\bar{j} \geq j_{0}$. Thus by Proposition 11.3 in [Giu84], for $H_{k}$-almost all $x_{0} \in \tilde{S}_{v}^{\bar{j}}$, we have

$$
\limsup _{r \rightarrow 0} \frac{H_{k}^{\infty}\left(\tilde{S}_{v}^{\bar{j}} \cap B\left(x_{0}, r\right)\right)}{\omega_{k} r^{k}} \geq 2^{-k} .
$$


Fix such an $x_{0}$, which we assume, without loss of generality, to be 0 . Choose a sequence $r_{n} \rightarrow 0$ such that for some $\epsilon>0$,

$$
\frac{H_{k}^{\infty}\left(\tilde{S}_{v}^{\bar{j}} \cap B_{r_{n}}\right)}{\omega_{k} r_{n}^{k}} \geq 2^{-k}-\epsilon .
$$

Consider $v_{n}(x)=v\left(r_{n} x\right) / S\left(r_{n}\right)$ and let $\bar{v}(x)$ be a blow-up limit of a subsequence of $v_{n}$, in the sense of Theorem 3.1 Fix a compact set $K$ in $B_{1}$ and $U$ open $\subset B_{1}$ with $U \supset K \cap \tilde{S}_{\bar{v}}^{\bar{j}}$. Assume that $x_{n} \in \tilde{S}_{v_{n}}^{j}, x_{n} \in K \backslash U$ and after passing to a subsequence, assume that $x_{n} \rightarrow \bar{x} \in K \backslash U$. Then $v_{n}\left(x_{n}\right) \rightarrow \bar{v}(\bar{x})$ and $\nabla v_{n}\left(x_{n}\right) \rightarrow \nabla \bar{v}(\bar{x})$, so that $\bar{x} \in S_{\bar{v}}$. Also, fix $0<r<1 / \bar{j}$. Then

$$
|\{\bar{v} \leq 0\} \cap B(\bar{x}, r)|=|\{\bar{v}<0\} \cap B(\bar{x}, r)|=\lim _{n \rightarrow \infty}\left|\left\{v_{n}<0\right\} \cap B\left(x_{n}, r\right)\right| \geq c_{0} r^{n},
$$

and so $\bar{x} \in \tilde{S}_{\bar{v}}^{\bar{j}}$, but $\bar{x} \in K \backslash U$ and $K \cap S_{\bar{v}}^{\bar{j}} \subset U$, which is a contradiction. Thus, we have shown that there exists $n_{0}$ so that, for $n>n_{0}$,

$$
U \supset K \cap \tilde{S}_{v_{n}}^{\bar{j}} .
$$

Then the proof of Lemma 11.5 in [Giu84] shows that for all $K \Subset B_{1}$,

$$
H_{k}^{\infty}\left(K \cap \tilde{S}_{\bar{v}}^{\bar{j}}\right) \geq \limsup _{n \rightarrow \infty} H_{k}^{\infty}\left(K \cap \tilde{S}_{v_{n}}^{\bar{j}}\right) .
$$

We next claim that

$$
\left\{x / r_{n}: x \in \tilde{S}_{v}^{j}\right\} \subset \tilde{S}_{v_{r_{n}}}^{\tilde{j}} .
$$

In fact, clearly $v_{n}\left(x / r_{n}\right)=0$ and $\nabla v_{n}\left(x / r_{n}\right)=0$. Consider now $\left\{y: v_{n}(y)<0\right\} \cap$ $B\left(x / r_{n}, r\right), 0<r<1 / \bar{j}$. This equals $\left\{y: v_{n}(y)<0\right\} \cap\left\{y:\left|y-x / r_{n}\right|<r\right\}$. By the transformation $y=z / r_{n}$, this set equals

$$
\{z: v(z)<0\} \cap\left\{z:\left|\frac{z}{r_{n}}-\frac{x}{r_{n}}\right|<r\right\}=\{z: v(z)<0\} \cap\left\{z:|z-x|<r r_{n}\right\} .
$$

Also, if $0<r<1 / \bar{j}$ then $r r_{n}<1 / \bar{j}$ for $n$ large. The Lebesgue measure of the set of $y$ 's equals $r_{n}^{-n}$ times the Lebesgue measure of the set of $z$ 's, which is then greater than $r_{n}^{-n} \cdot c_{0}\left(r r_{n}\right)^{n}=c_{0} r^{n}$, so that $x / r_{n} \in \tilde{S}_{v_{n}}^{\bar{j}}$. But then

$$
H_{k}^{\infty}\left(B_{1} \cap \tilde{S}_{v_{n}}^{\bar{j}}\right) \geq \frac{H_{k}^{\infty}\left(B_{r_{n}} \cap \tilde{S}_{v}^{\bar{j}}\right)}{\omega_{k} r_{n}^{k}} \geq 2^{-k}-\epsilon,
$$

by our choice of $r_{n}$. Hence, using (3.16), we see that

$$
H_{k}^{\infty}\left(B_{1} \cap \tilde{S}_{\bar{v}}^{\bar{j}}\right)>0 .
$$

We now consider our classification of blow-ups. If $\lim _{r \downarrow 0} W_{1}(r)=-\infty$, then, by (ii), $\bar{v}$ is a non-zero, harmonic polynomial homogeneous of degree 2 . But then, as 
is well-known, $H_{n-2}\left(S_{\bar{v}}\right)<\infty, S_{\bar{v}} \supset \tilde{S}_{\bar{v}}^{\bar{j}}$, which contradicts 3.18 since $k>n-2$. If $\lim _{r \downarrow 0} W_{1}(r)>-\infty$, then in view of Theorem 3.1 i) and Lemma 3.8, after passing to a further subsequence, we can assume that $r_{n}^{2} / S\left(r_{n}\right) \rightarrow \alpha \in(0, \infty)$. Hence $\alpha \bar{v}=\bar{v}_{1}$, where $\bar{v}_{1}$ is a solution to $(3.2)$ homogeneous of degree 2 with $f=f_{0}, g=g_{0}$ both constants. We can now do the dimension reduction. From (3.18), we know that $H_{k}^{\infty}\left(B_{1} \cap \tilde{S}_{\bar{v}}^{\bar{j}}\right)>0$. Using Lemmas 11.2 and 11.3 of [Giu84], we can find $\bar{x} \in \tilde{S}_{\bar{v}}^{\bar{j}} \backslash\{0\}$ such that

$$
\lim _{r \rightarrow 0} \frac{H_{k}^{\infty}\left(\tilde{S}_{\bar{v}}^{\bar{j}} \cap B(\bar{x}, r)\right)}{\omega_{k} r^{k}} \geq 2^{-k} .
$$

By homogeneity of $\bar{v}_{1}$, we can assume that $\bar{x} \in \partial B_{1}$. We can pick a sequence $r_{n} \rightarrow 0$, and consider a blow-up limit $\bar{v}_{1,0}$ at $\bar{x}$ with respect to $r_{n}$. By the homogeneity of $\bar{v}_{1}$, it is easy to see that $\bar{v}_{1,0}$ is constant in the $\bar{x}$ direction. After rotation, we can assume this direction to be the $x_{n}$ direction. But it is easy to see that $\left(x_{1}, \ldots, x_{n-1}, x_{n}\right) \in \tilde{S}_{\bar{v}_{1,0} \mid \mathbb{R}^{n-1}}^{\bar{j}}$ and that $H_{k-1}\left(\tilde{S}_{\bar{v}_{1,0} \mid \mathbb{R}^{n-1}}^{\bar{j}}\right)>0$. Proceeding in this way $n-2$ times, we find a contradiction to Theorem 3.2. which concludes the proof.

We are now ready to establish partial $C^{1,1}$ bounds.

Definition 3.16. Let $f$ be a $C^{1, \gamma}$ function, $0 \leq \gamma<1$, defined in a neighborhood of a point $x_{0}$. We say that $f$ satisfies $C^{1,1}$ bounds at $x_{0}$ if

$$
\lim _{r \rightarrow 0} \sup _{\left|x-x_{0}\right| \leq r} \frac{\left|f(x)-\left(x-x_{0}\right) \nabla f\left(x_{0}\right)-f\left(x_{0}\right)\right|}{r^{2}}<+\infty .
$$

We call the above limit the $C^{1,1}$ norm of $f$ at $x_{0}$.

Our next task is to show that our solutions $v$ satisfy $C^{1,1}$ bounds at all $x_{0} \in \mathcal{F}$, except for a set of Hausdorff dimension at most $n-2$. We start out with some preliminary results.

Lemma 3.17. There exists a constant $c_{n}$ such that for all harmonic polynomials $p$ homogeneous of degree 2 with $p \not \equiv 0$, we have

$$
\left|\{p<0\} \cap B_{1}\right| \geq c_{n} .
$$

Proof. We can assume $\int_{B_{1}} p^{2}=1$. If the conclusion fails, we can find a sequence $p_{j}$ such that $\int_{B_{1}} p_{j}^{2}=1$ and $p_{j}$ is a harmonic polynomial homogeneous of degree 2 with $\left|\left\{p_{j}<0\right\} \cap B_{1}\right| \rightarrow 0$ as $j \rightarrow 0$. After passing to a subsequence, $p_{j} \rightarrow p_{0}$ where $p_{0}$ is a harmonic polynomial homogeneous of degree $2, \int_{B_{1}} p_{0}=1$ and $\left|\left\{p_{0}<0\right\} \cap B_{1}\right|=0$. By homogeneity, $p_{0} \geq 0$, but $p_{0}(0)=0$, so that $p_{0} \equiv 0$, a contradiction.

Lemma 3.18. Let $c_{n}$ be as in Lemma 3.17. Assume that $v$ is a solution and $x_{0} \in S_{v}$. Assume that $\sup _{\left|x-x_{0}\right|<r_{j}}|v(x)| / r_{j}^{2} \rightarrow \infty$ for some sequence $r_{j} \rightarrow 0$. Then

$$
\left|\{v<0\} \cap B\left(x_{0}, r\right)\right| \geq \frac{c_{n}}{2} r^{n} \quad \text { for } 0<r<r_{0}\left(x_{0}\right) .
$$


Proof. If not, there exists $\tilde{r}_{j} \rightarrow 0$ such that

$$
\left|\{v<0\} \cap B\left(x_{0}, \tilde{r}_{j}\right)\right|<\frac{c_{n}}{2} \tilde{r}_{j}^{n} .
$$

But, by the proof of Corollary $3.13, S\left(2 \tilde{r}_{j}\right) /\left(2 \tilde{r}_{j}\right)^{2} \rightarrow+\infty$. By Corollary $3.14, S\left(\tilde{r}_{j}\right) / \tilde{r}_{j}^{2}$ $\rightarrow+\infty$. Then, by Theorem 3.1(ii), $v\left(\tilde{r}_{j} x+x_{0}\right) / S\left(\tilde{r}_{j}\right)$ converges, after passing to a subsequence, to a $\bar{w}$ which is a non-zero harmonic polynomial homogeneous of degree 2 . But then $\left|\{\bar{w}<0\} \cap B_{1}\right| \leq c_{n} / 2$, which contradicts Lemma 3.17

Theorem 3.4 (Pointwise $C^{1,1}$ bounds on $S_{v}$ ). Let $v$ be a solution. Then the Hausdorff dimension of the set $B_{v}=\left\{x_{0} \in S_{v}: v\right.$ does not have pointwise $C^{1,1}$ bounds at $\left.x_{0}\right\}$ is at most $n-2$.

Proof. Combine Lemma 3.18 with Theorem 3.3

Remark 3.19. If $x_{0} \in \mathcal{F}$ and $\nabla v\left(x_{0}\right) \not \equiv 0$, then by [CGK00], $\mathcal{F}$ is real-analytic in a neighborhood of $x_{0}$ and by boundary elliptic regularity we obtain $C^{1,1}$ bounds at $x_{0}$. Thus, the set of points in $\mathcal{F}$ for which $v$ does have pointwise $C^{1,1}$ bounds has Hausdorff dimension at most $n-2$.

Remark 3.20. The results in Theorems 3.2 3.4 and in Remark 3.19 are sharp. We show this for the case $f=f_{0}, g=g_{0}$ constants. We first make some preliminary comments in the case $n=2$. In this case, Blank ([Bla04] $)$ found all solutions homogeneous of degree 2 for which $\{v<0\} \neq \varnothing$. The calculation in Appendix 2 shows that, for these solutions, $W(1)>-A$, where $A$ depends only on $f_{0}, g_{0}$. Shahgholian ([Sha]) observed that there are other solutions homogeneous of degree 2 , which are non-negative. In fact, any such solution $\bar{v}$ satisfies $\Delta \bar{v}=f_{0}, \bar{v} \geq 0$ in $\mathbb{R}^{2}$. Let $w=\bar{v}-\left(f_{0} / 4\right)\left(x_{1}^{2}+x_{2}^{2}\right)$. This is a harmonic polynomial homogeneous of degree 2 , so that after rotation $w=a\left(x_{1}^{2}-x_{2}^{2}\right)$ or

$$
\bar{v}=\left(a+\frac{f_{0}}{4}\right) x_{1}^{2}+\left(\frac{f_{0}}{4}-a\right) x_{2}^{2}
$$

Since $\bar{v} \geq 0$, we must have $-f_{0} / 4 \leq a \leq f_{0} / 4$. For those solutions we also find $W(1)>$ $-A, A$ depending only on $f_{0}, g_{0}$. Combining these comments with Theorem 3.1 we see that for $n=2$ there exists $A=A\left(f_{0}, g_{0}\right)$ such that if for $v$ we have $\lim _{r \downarrow 0} W_{1}(r)<-A$, then $\lim _{r \downarrow 0} W_{1}(r)=-\infty$ and $\lim _{r \downarrow 0} S(r) / r^{2}=+\infty$. One can then use the argument in [AW06] to see that by the Andersson-Weiss construction we can find solutions (taking $M$ large in [AW06]) so that $W_{1}(1)<-A$, and hence solutions which do not have $C^{1,1}$ bounds in any neighborhood of 0 . In light of Lemma 3.17, this shows the sharpness of Theorem 3.2 and of Theorem 3.4 when $n=2$. To create higher-dimensional examples, one just adds $n-2$ dummy variables. It remains a challenging problem to see if such pathology can hold for solutions of 3.2.

We now turn to the issue of uniform pointwise $C^{1,1}$ bounds. 
Theorem 3.5. Let $S_{v}^{(1)}=S_{v} / S_{v}^{(2)}$, where

$$
\begin{aligned}
S_{v}^{(2), j} & =\left\{x_{0} \in S_{v}:|\{v<0\} \cap B(x, r)| \geq r^{n} / j, 0<r<r_{0, j}\left(x_{0}\right)\right\}, \\
S_{v}^{(2)} & =\bigcup_{j=1}^{\infty} S_{v}^{(2), j} .
\end{aligned}
$$

By Theorem 3.3 the Hausdorff dimension of $S_{v}^{(2)}$ is at most $n-2$. Then for $x_{0} \in S_{v}^{(1)}$ we have uniform $C^{1,1}$ estimates, i.e. there exists $C=C\left(\tilde{B}_{1}, \tilde{B}_{2}, \tilde{B}_{3}, n, \eta_{0}, r_{0}, N\right)>0$ such that for all $x_{0} \in S_{v}^{(1)}$,

$$
\sup _{\substack{\left|x-x_{0}\right| \leq r \\ 0<r<r_{0} / 2}} \frac{|v(x)|}{r^{2}} \leq C .
$$

Proof. In light of Theorem 3.1, Lemma 3.17 and Corollary 3.13 it suffices to show that for such $x_{0}, \lim _{r \downarrow 0} W_{1}(r)>-A$, where $A$ has the right dependence. Let $\bar{v}$ be a blow-up limit at such an $x_{0}$. Clearly, $\bar{v} \geq 0$. Thus, it suffices to show that, for such $\bar{v}, W(1, \bar{v})>-A$. But $\Delta \bar{v}=f_{0}$ and

$$
\int_{B_{1}} \Delta \bar{v}=\omega_{n} f_{0}=\int_{\partial B_{1}} \frac{\partial \bar{v}}{\partial v}=2 \int_{\partial B_{1}} \bar{v}
$$

since $\bar{v}$ is homogeneous of degree 2. Thus, $\int_{\partial B_{1}} \bar{v}=\omega_{n} f_{0} / 2$. Since $\bar{v}$ is non-negative and subharmonic, $\int_{B_{1}} \bar{v} \leq c_{n} f_{0} \omega_{n} / 2$. The rest of the proof follows easily from interior estimates and homogeneity.

Remark 3.21. Similarly, if $K \Subset\left\{x_{0} \in \mathcal{F}: \nabla v\left(x_{0}\right) \neq 0\right\}$ we also have uniform pointwise $C^{1,1}$ bounds on $K$. (See Remark 3.19.)

Our final result is a partial regularity result for $\mathcal{F}$.

Theorem 3.6. Let $v$ be a solution of (3.2) satisfying our assumptions. Then $\mathcal{F}=\mathcal{F}_{0} \cup$ $S_{v}^{(1)} \cup S_{v}^{(2)}$, where $S_{v}^{(2)}$ has Hausdorff dimension at most $n-2, S_{v}^{(1)}$ is $(n-1)$-regular, i.e. $H_{n-1}\left(S_{v}^{(1)}\right) \leq C=C\left(\tilde{B}_{1}, \tilde{B}_{2}, \tilde{B}_{3}, N, \eta_{0}, r_{0}, n\right), \mathcal{F}_{0}$ is relatively open, and for each $x_{0} \in \mathcal{F}$ there exists a neighborhood $U_{x_{0}}$ such that $\mathcal{F} \cap U_{x_{0}}$ is a real-analytic hypersurface.

Proof. $\mathcal{F}_{0}=\left\{x_{0} \in \mathcal{F}: \nabla v\left(x_{0}\right) \neq 0\right\}$ and $S_{v}^{(1)}, S_{v}^{(2)}$ are defined in Theorem 3.5 . From Theorem 3.5 we know that the Hausdorff dimension of $S_{v}^{(2)}$ is at most $n-2$, so it remains to show that $S_{v}^{(1)}$ is $(n-1)$-regular (in light of Theorem 8 in [CGK00], which shows the desired property of $\mathcal{F}_{0}$ ). In order to show this, we make some preliminary claims.

Claim 3.22. If $x_{0} \in S_{v}^{(1)}$ (without loss of generality, we take $\left.x_{0}=0\right)$ then $|\nabla v(x)| \leq C r$ for $0<r<r_{0} / 4$ and $x \in B_{r}$, with $C$ as in the statement of Theorem 3.6

In order to establish the claim, note that $|v(x)| \leq C|x|^{2}$ for $x \in B_{2 r}$, by Theorem 3.5 Next, we use Lemma 3.6(ii), (iii) to obtain

$$
\int_{B_{r}}\left|\nabla v^{+}\right|^{2} \leq c_{n} C r^{n+2} \text { and } \int_{B_{r}}\left|\nabla v^{-}\right|^{2} \leq c_{n}\left\{C+a_{2}\right\} r^{n+2} \text {, }
$$


so that $\int_{B_{r}}|\nabla v|^{2} \leq c_{n}\left(C+a_{2}\right) r^{n+2}$. Next, consider $v_{r}$ on $B_{1}$. We have $\int_{B_{1}}\left|v_{r}\right|^{2} \leq C$, $\int_{B_{1}}\left|\nabla v_{r}\right|^{2} \leq C$, and $\left|\Delta v_{r}\right| \leq C$. From this it is easy to see that $\left|\nabla v_{r}\right| \leq C$ for $|x| \leq 1 / 2$, which is our claim.

The next step is:

Claim 3.23. Let $x_{0} \in S_{v}$, let $e_{i}$ be a fixed coordinate direction, and set $v_{e_{i}}=e_{i} \cdot \nabla v$. Then, for $h \geq 0$ small,

$$
\int_{B\left(x_{0}, r_{0} / 2\right) \cap\{x:|\nabla v| \leq h\}}\left|\nabla v_{e_{i}}\right|^{2} \leq C h .
$$

To establish Claim 3.23. we first introduce a truncation $\bar{v}_{e_{i}}$ of $v_{e_{i}} \in W^{1,2}(U) \cap C^{\gamma}(\bar{U})$, where

$$
\bar{v}_{e_{i}}= \begin{cases}v_{e_{i}} & \text { if }-h<v_{e_{i}}<-\delta \text { or } \delta<v_{e_{i}}<h, \\ 0 & \text { if }\left|v_{e_{i}}\right| \leq \delta, \\ h & \text { if }\left|v_{e_{i}}\right| \geq h .\end{cases}
$$

Let $\psi$ be a standard mollifier and for $0<\epsilon \ll \delta$, consider the mollifier $v_{e_{i}} * \psi_{\epsilon}$. We will apply Green's theorem to

$$
\int_{B_{r}} \nabla \bar{v}_{e_{i}} \cdot \nabla\left(v_{e_{i}} * \psi_{\epsilon}\right) \quad \text { for } r_{0} / 2<r<r_{0},
$$

where we have assumed that $x_{0}=0$. Since $|\mathcal{F}|=0$ (see Theorem 1.1 . c)), this integral equals

$$
\int_{B_{r} \cap\{v>0\}} \nabla \bar{v}_{e_{i}} \cdot \nabla\left(v_{e_{i}} * \psi_{\epsilon}\right)+\int_{B_{r} \cap\{v<0\}} \nabla \bar{v}_{e_{i}} \cdot \nabla\left(v_{e_{i}} * \psi_{\epsilon}\right) .
$$

On $S_{v}, \nabla v=0$, so that $\bar{v}_{e_{i}}$ will vanish on a neighborhood of $S_{v}$. In fact, if $\left|v_{e_{i}}(x)\right| \geq \delta$ and $z_{0} \in S_{v}$, then $\delta \leq\left|v_{e_{i}}(x)-v_{e_{i}}\left(z_{0}\right)\right| \leq C\left|x-z_{0}\right|^{\gamma}$. In $\mathcal{F} \backslash \operatorname{nbd}\left(S_{v}\right)$, we have analyticity of $\mathcal{F}$ and a well-defined normal, so that we can integrate by parts in the above integrals, using Green's theorem, to obtain for the above sum the expression

$$
\begin{array}{r}
-\int_{B_{r} \cap\{v>0\}} \bar{v}_{e_{i}} \Delta\left(v_{e_{i}} * \psi_{\epsilon}\right)-\int_{B_{r} \cap\{v<0\}} \bar{v}_{e_{i}} \Delta\left(v_{e_{i}} * \psi_{\epsilon}\right)+\int_{\partial B_{r}} \bar{v}_{e_{i}} \frac{\partial}{\partial v}\left(v_{e_{i}} * \psi_{\epsilon}\right) \\
+\int_{B_{r} \cap \partial\{v>0\}} \bar{v}_{e_{i}} \frac{\partial}{\partial v}\left(v_{e_{i}} * \psi_{\epsilon}\right)+\int_{B_{r} \cap \partial\{v<0\}} \bar{v}_{e_{i}} \frac{\partial}{\partial v}\left(v_{e_{i}} * \psi_{\epsilon}\right)
\end{array}
$$

The last two integrals cancel each other since the normals point in opposite directions, in pieces of a real-analytic surface. Thus, we have obtained

$$
\begin{aligned}
\int_{B_{r}} \nabla \bar{v}_{e_{i}} \cdot \nabla\left(v_{e_{i}} * \psi_{\epsilon}\right)= & -\int_{B_{r} \cap\{v>0\}} \bar{v}_{e_{i}} \Delta\left(v_{e_{i}} * \psi_{\epsilon}\right) \\
& -\int_{B_{r} \cap\{v<0\}} \bar{v}_{e_{i}} \Delta\left(v_{e_{i}} * \psi_{\epsilon}\right)+\int_{\partial B_{r}} \bar{v}_{e_{i}} \frac{\partial}{\partial v}\left(v_{e_{i}} * \psi_{\epsilon}\right) .
\end{aligned}
$$


We next average this identity over $r \in\left(r_{0} / 2,3 r_{0} / 4\right)$. We first estimate the averaged last term. Its absolute value is bounded by

$$
c_{n} h \int_{r_{0} / 2 \leq|x| \leq 3 r_{0} / 4}\left|\nabla v_{e_{i}} * \psi_{\epsilon}\right| \leq c_{n} C h .
$$

We next consider the absolute value of the averaged left hand side as $\epsilon \rightarrow 0$. It converges to

$$
\left|\frac{4}{r_{0}} \int_{r_{0} / 2}^{3 r_{0} / 4} \int_{B_{r}} \nabla \bar{v}_{e_{i}} \cdot \nabla v_{e_{i}}\right| \underset{\delta \rightarrow 0}{\longrightarrow}\left|\frac{4}{r_{0}} \int_{r_{0} / 2}^{3 r_{0} / 4} \int_{B_{r}} \nabla \tilde{v}_{e_{i}} \cdot \nabla v_{e_{i}}\right|,
$$

where

$$
\tilde{v}_{e_{i}}= \begin{cases}v_{e_{i}} & \text { if }\left|v_{e_{i}}\right| \leq h \\ h & \text { otherwise }\end{cases}
$$

This last expression is bounded from below by $c_{n} \int_{B_{r_{0} / 2}}\left|\nabla \tilde{v}_{e_{i}}\right|^{2}$.

The absolute value of the sum of the averaged first two terms on the right hand side converges (upon letting first $\epsilon \rightarrow 0$ and then $\delta \rightarrow 0$ ) to

$$
\left|\frac{4}{r_{0}} \int_{r_{0} / 2}^{3 r_{0} / 4} \int_{B_{r} \cap\{v>0\}} \tilde{v}_{e_{i}} \Delta v_{e_{i}}+\frac{4}{r_{0}} \int_{r_{0} / 2}^{3 r_{0} / 4} \int_{B_{r} \cap\{v<0\}} \tilde{v}_{e_{i}} \Delta v_{e_{i}}\right| .
$$

But on $\{v>0\}, \Delta v_{e_{i}}=\partial_{e_{i}} f$, and on $\{v<0\}, \Delta v_{e_{i}}=-\partial_{e_{i}} g$. Hence, the above sum is bounded by

$$
h\left(\int_{B_{r_{0}} \cap\{v>0\}}\left|\Delta v_{e_{i}}\right|+\int_{B_{r_{0}} \cap\{v<0\}}\left|\Delta v_{e_{i}}\right|\right) \leq C h .
$$

Finally, gathering terms and using the fact that

$$
\int_{B_{r_{0} / 2}}\left|\nabla \tilde{v}_{e_{i}}\right|^{2}=\int_{B_{r_{0} / 2} \cap\left\{\left|v_{e_{i}}\right| \leq h\right\}}\left|\nabla v_{e_{i}}\right|^{2},
$$

we obtain Claim 3.23

We next complete the proof of the bound $H_{n-1}\left(S_{v}^{(1)}\right) \leq C$. Fix $z_{0} \in S_{v}^{(1)}$. It suffices to prove our bound for $S_{v}^{(1)} \cap B\left(z_{0}, r_{0} / 4\right)$. Consider the cover of $S_{v}^{(1)} \cap B\left(z_{0}, r_{0} / 4\right)$ by the balls $B\left(x_{0}, r\right)$ with $x_{0}$ in $S_{v}^{(1)} \cap B\left(z_{0}, r_{0} / 4\right)$ and $0<r<r_{0} / 100$. It has a finite subcover, and by the Vitali covering lemma, we can find $r$ and $\tilde{N}$ disjoint balls $B\left(x_{i}, r\right)$ with $x_{i} \in S_{v}^{(1)} \cap B\left(z_{0}, r_{0} / 4\right)$ so that $S_{v}^{(1)} \cap B\left(z_{0}, r_{0} / 4\right) \subset \bigcup_{i=1}^{\tilde{N}} B\left(x_{i}, 5 r\right)$. The disjointness of $\left\{B\left(x_{i}, r\right)\right\}$ gives $\sum_{i=1}^{\tilde{N}} \chi_{B\left(x_{i}, 5 r\right)}(x) \leq c_{n}$. By Claim $3.22|\nabla v(x)| \leq C r$ in $B\left(x_{i}, 5 r\right)$. By $[3.2,,|\Delta v| \geq C$. We then have

$$
\begin{aligned}
c_{n} \tilde{N} c r^{n} & \leq \sum_{i} \int_{B\left(x_{i}, 5 r\right)}(\Delta v)^{2} \leq \int_{\{|\nabla v(x)| \leq c r\}}(\Delta v)^{2} \sum_{i=1}^{\tilde{N}} \chi_{B\left(x_{i}, 5 r\right)} \\
& \leq c_{n} \int_{B\left(z_{0}, r_{0} / 2\right) \cap\{|\nabla v(x)| \leq c r\}}(\Delta v)^{2} \leq C r,
\end{aligned}
$$

by Claim 3.23 Thus, $\tilde{N} r^{n-1} \leq C$, which gives our Hausdorff measure bound. 
To conclude this paper we give a simple result in the direction of showing that better regularity results can hold for solutions of the composite problem than for solutions of (3.2) (see the end of Remark 3.20). We will prove that geometric assumptions on $\Omega$ can ensure that for all solutions of the composite problem, $S_{u}=\emptyset$ and thus $\mathcal{F}$ is real-analytic and $u$ is $C^{1,1}$.

Proposition 3.7. Let $\Omega \subset \mathbb{R}^{2}$ have two axes of symmetry. Then for all solutions $u$ of the composite problem (1.1), 1.2) we have $S_{u}=\emptyset$ and hence $\mathcal{F}$ is real-analytic and $u \in C^{1,1}$.

Proof. We recall (see $\left[\mathrm{CGI}^{+} 00\right]$ ) that we say that $\Omega$ has an axis of symmetry $L$ (which we take to be $\left.\left\{x_{1}=0\right\}\right)$ if whenever $\left(x_{1}, x_{2}\right)$ belongs to $\Omega$, so does $\left(-x_{1}, x_{2}\right)$ and the set $\left\{x_{1}:\left(x_{1}, x_{2}\right) \in \Omega\right\}$ is either $\emptyset$ or an interval $(-c, c)$ for each $x_{2}$. Let us give the proof, for simplicity, in the case when the two axes $L_{1}, L_{2}$ are the $x_{1}$ - and $x_{2}$-axis. It is shown in $\left[\mathrm{CGI}^{+} 00\right.$, Theorem 4] that any solution $u$ is symmetric with respect to $x_{1}$ (and $x_{2}$ ) and $u$ is strictly decreasing in $x_{1}$, for $x_{1} \geq 0$ (in $x_{2}$, for $x_{2} \geq 0$ ). (The strict decrease follows from $\alpha<\Lambda$, see $\left[\mathrm{CGI}^{+} 00\right.$, bottom of p. 326]). Because of the strict decrease, $\frac{\partial}{\partial x_{1}} u\left(x_{1}, x_{2}\right) \neq 0$ for $x_{1} \neq 0$ and $\frac{\partial}{\partial x_{2}} u\left(x_{1}, x_{2}\right) \neq 0$ for $x_{2} \neq 0$. Thus, the only possible point in $S_{u}$ is $(0,0)$. But, by the increase and decrease described before, $u(0,0)=\sup _{\Omega} u$. Recall that $D=\{0 \leq u \leq c\}$ and $\mathcal{F}=\{u=c\}$. If $c=\sup _{\Omega} u$ then $D=\Omega$, which contradicts $|D|=A<|\Omega|$. Thus, $(0,0) \notin \mathcal{F}$ and the proposition follows.

\section{Appendix I}

The results A1.9, A1.10, below can be found in $[\mathrm{CP}]$. They are reproduced here for the reader's benefit.

We have the equation

$$
-\Delta u_{t}+\alpha \chi_{D_{t}} u_{t}=\lambda(t) u_{t}
$$

and the corresponding one for $u_{0}=u$, given by

$$
-\Delta u+\alpha \chi_{D} u=\lambda u
$$

where $\lambda(0)=\lambda$. We also note that by our definition of $D_{t}$,

$$
\chi_{D_{t}}(x)=\chi_{D}\left(\phi_{-t}(x)\right)
$$

We set

$$
V(x)=\left.\frac{d \phi_{t}(x)}{d t}\right|_{t=0}
$$

and assume that $V \in C^{2}(\Omega)$ and that $V$ is supported in a compact set $S$.

Multiplying A1.1 by $u, \mathrm{~A} 1.2$ by $u_{t}$ and subtracting we get

$$
u_{t} \Delta u-u \Delta u_{t}+\alpha\left(\chi_{D}\left(\phi_{-t}(x)\right)-\chi_{D}(x)\right) u u_{t}=(\lambda(t)-\lambda) u u_{t} .
$$


We integrate A1.4 over $\Omega$. Since $u=u_{t}=0$ on $\partial \Omega$, we get

$$
\int_{\Omega}\left[u_{t} \Delta u-u \Delta u_{t}\right]=0
$$

Thus the integral over $\Omega$ of $\mathrm{A} 1.4$ becomes

$$
\int_{\Omega} \alpha\left(\chi_{D}\left(\phi_{-t}(x)\right)-\chi_{D}(x)\right) u u_{t}=(\lambda(t)-\lambda) \int_{\Omega} u u_{t} .
$$

Now from A1.1 we notice that if we normalize our functions: $\left\|u_{t}\right\|_{2}=1$, as we certainly can, we always have $\left\|u_{t}\right\|_{2,2} \leq C$. Now,

$$
\left|\int_{\Omega}\left(u u_{t}-u^{2}\right)\right| \leq \int_{\Omega} u\left|u-u_{t}\right|
$$

In a tubular neighborhood $\mathcal{U}$ of $\partial \Omega$ we have

$$
\int_{\mathcal{U}} u\left|u-u_{t}\right| \leq C\left(\int_{\mathcal{U}} u^{2}\right)^{1 / 2} \leq \epsilon
$$

Outside $\mathcal{U}$ by the uniform $W^{2,2}$ bounds of $u_{t}$ we have strong convergence of $u_{t}$ to $u$ in $L^{2}$, and

$$
\lim _{t \rightarrow 0} \int_{\Omega} u u_{t}=\int_{\Omega} u^{2}=1
$$

Now we change variables in the left side of A1.5. We set $\phi_{-t}(x)=y$. Thus, $x=$ $\phi_{-t}^{-1}(y)$, and the left side of $\mathrm{A} 1.5$ becomes

$$
\alpha \int_{\Omega} \chi_{D}(x)\left(h_{t}\left(\phi_{-t}^{-1}(x)\right) J_{t}(x)-h_{t}(x)\right) d x .
$$

Here we have set $h_{t}=u u_{t}$ and $J_{t}(x)$ is the Jacobian of the transformation $y=\phi_{-t}^{-1}(x)$. Since $\phi_{0}$ is the identity, it is well-known that

$$
J_{t}(x)=1+t \operatorname{div} V+O\left(t^{2}\right) .
$$

See for example Lemma 1 (p. 69) in [Arn97]; in fact (A1.7]) is an elementary consequence of the fact that for an $n \times n$ matrix $B, \operatorname{det}(I-t B)^{-1}=1+t \operatorname{trace}(B)+O\left(t^{2}\right)$. Since $h_{t} \in C^{1, \beta}$, we see that

$$
h_{t}\left(\phi_{-t}^{-1}(x)\right) J_{t}(x)-h_{t}(x)=t\left((V \cdot \nabla) h_{t}(x)+h_{t}(x) \operatorname{div} V\right)+o(t) .
$$

Thus on division by $t$ and letting $t \rightarrow 0$ we see easily that

$$
\lim _{t \rightarrow 0} \frac{h_{t}\left(\phi_{-t}^{-1}(x)\right) J_{t}(x)-h_{t}(x)}{t}=(V \cdot \nabla)\left(u^{2}\right)+u^{2} \operatorname{div} V .
$$


The term on the right above is $\operatorname{div}\left(V u^{2}\right)$. Thus dividing A1.5 by $t$ and using A1.6 we easily get

$$
\lambda^{\prime}(0)=\alpha \int_{D} \operatorname{div}\left(V u^{2}\right)=\alpha \int_{D \cap S} \operatorname{div}\left(V u^{2}\right) .
$$

By the hypothesis that the part of the boundary of $\partial D$ that lies inside the support of $V$ is regular enough to have a bona fide unit outer normal $v$, and Green's theorem, the last integral above yields

$$
\lambda^{\prime}(0)=\alpha \int_{S \cap \partial D}\langle V, v\rangle u^{2}
$$

Now consider

$$
\left|D_{t}\right|-|D|=\int_{\Omega}\left(\chi_{D}\left(\phi_{-t}(x)\right)-\chi_{D}(x)\right) d x .
$$

Change variables in the integral above as before to get

$$
\int_{D}\left(J_{t}(x)-1\right) d x
$$

By A1.7 again we see the integral above is

$$
t \int_{D} \operatorname{div} V d x+O\left(t^{2}\right)
$$

Thus we easily get

$$
\left.\frac{d}{d t}\left|D_{t}\right|\right|_{t=0}=\int_{D} \operatorname{div} V d x=\int_{S \cap \partial D}\langle V, v\rangle d \sigma .
$$

If $u=c$ along $\partial D$, combining A1.8 and A1.9 we get

$$
\lambda^{\prime}(0)=\left.\alpha c^{2} \frac{d}{d t}\left|D_{t}\right|\right|_{t=0}=\alpha c^{2} \int_{\partial D}\langle V, v\rangle d \sigma
$$

\section{Appendix II}

We now prove the assertions made in Remark 3.20. We use Blank's [Bla04] notation. We have

$$
f_{1}(\theta)=C_{+} \sin \left(2 \theta+D_{+}\right)+\gamma, \quad f_{1}>0,
$$

and also

$$
f_{2}(\theta)=C_{-} \sin \left(2 \theta+D_{-}\right)+\mu, \quad f_{2}<0 .
$$

Now we focus on the interval $[0,2 \pi / 3]$. First for $\theta_{0} \in(0,2 \pi / 3)$, we know $f_{1}\left(\theta_{0}\right)=$ $f_{2}\left(\theta_{0}\right)=0$ and $f_{1}^{\prime}\left(\theta_{0}\right)=f_{2}^{\prime}\left(\theta_{0}\right)=0$. We get

$$
C_{+} \sin \left(2 \theta_{0}+D_{+}\right)+\gamma=C_{-} \sin \left(2 \theta_{0}+D_{-}\right)+\mu=0
$$


and

$$
C_{+} \cos \left(2 \theta_{0}+D_{+}\right)=C_{-} \cos \left(2 \theta_{0}+D_{-}\right) .
$$

After squaring and adding both equations, this leads to

$$
C_{+}^{2}-C_{-}^{2}=\gamma^{2}-\mu^{2} \text {. }
$$

Next because $f_{1}(0)=f_{1}\left(\theta_{0}\right)=0$, we get

$$
C_{+} \sin \left(D_{+}\right)=-\gamma, \quad D_{+}=\arcsin \left(-\gamma / C_{+}\right)
$$

and so

$$
\theta_{0}=\pi / 2+\arcsin \left(\gamma / C_{+}\right) .
$$

Since $f_{2}\left(\theta_{0}\right)=0$, inserting the value of $\theta_{0}$ from $\mathrm{A} 2.3$ in the expression for $f_{2}$, we see that

$$
D_{-}=\arcsin \left(\mu / C_{-}\right)-2 \arcsin \left(\gamma / C_{+}\right) .
$$

Lastly, $f_{2}(2 \pi / 3)=0$, so

$$
C_{-} \sin \left(4 \pi / 3+D_{-}\right)=-\mu
$$

and we get

$$
C_{-}=\mu / \sin \left(\pi / 3+D_{-}\right) .
$$

Now assume $\left|C_{+}\right|>10^{6}(|\gamma|+|\mu|)$. Then $\left|C_{-}\right|>10^{6}(|\gamma|+|\mu|)$ by A2.1). Thus, $\left|D_{-}\right| \leq \pi / 20$ from $\mathrm{A} 2.4$. From $\mathrm{A} 2.5$ we get

$$
\left|C_{-}\right| \leq 2|\mu|,
$$

and we get a bound on $\left|C_{+}\right|$from $\mathrm{A} 2.1$ again.

Acknowledgments. Research of S. Chanillo and C. E. Kenig was supported in part by NSF.

\section{References}

[Arn97] Arnold, V. I.: Mathematical Methods of Classical Mechanics. Grad. Texts in Math. 60, Springer (1997) Zbl 0386.70001 MR 1345386

[AW06] Andersson, J., Weiss, G. S.: Cross-shaped and degenerate singularities in an unstable elliptic free boundary problem. J. Differential Equations 228, 633-640 (2006) Zbl 05072807 MR 2289547

[Bla04] Blank, I.: Eliminating mixed asymptotics in obstacle type free boundary problems. Comm. Partial Differential Equations 29, 1167-1186 (2004) Zbl 1082.35165 MR 2097580

$\left[\mathrm{CGI}^{+}\right.$00] Chanillo, S., Grieser, D., Imai, M., Kurata, K., Ohnishi, I.: Symmetry breaking and other phenomena in the optimization of eigenvalues for composite membranes. Comm. Math. Phys. 214, 315-337 (2000) Zbl 0972.49030 MR 1796024

[CGK00] Chanillo, S., Grieser, D., Kurata, K.: The free boundary problem in the optimization of composite membranes. In: Contemp. Math. 268, Amer. Math. Soc., 61-81 (2000) Zbl 0988.35124 MR 1804790

[CP] Chanillo, S., Pedrosa, R.: Hadamard's formulae for composite membranes. Preprint. 
[Giu84] Giusti, E.: Minimal Surfaces and Functions of Bounded Variation. Monogr. Math. 80, Birkhäuser (1984) Zbl 0545.49018 MR 0775682

[GT83] Gilbarg, D., Trudinger, N.: Elliptic Partial Differential Equations of Second Order. Springer (1983) Zbl 0562.35001 MR 0737190

[Kat73] Kato, T.: Schrödinger operators with singular potentials. In: Proc. Int. Symposium on Partial Differential Equations and the Geometry of Normed Linear Spaces (Jerusalem, 1972), Israel J. Math. 13, 135-148 (1973) Zbl 0246.35025 MR 0333833

[MW07] Monneau, R., Weiss, G. S.: An unstable elliptic free boundary problem arising in solid combustion. Duke Math. J. 136, 321-341 (2007) Zbl 05129536 MR 2286633

[Sha] Shahgholian, H.: The singular set for the composite membrane problem. Comm. Math. Phys. 217, 93-101 (2007) MR 2283955

[Wei98] Weiss, G. S.: Partial regularity for weak solutions of an elliptic free boundary problem. Comm. Partial Differential Equations 23, 439-455 (1998) Zbl 0897.35017 MR 1620644 